\title{
Dynamic Structural Design of Offshore Direct-Drive Wind Turbine Electrical Generators
}

\author{
Pablo Jaen-Sola ${ }^{1}$, Alasdair S. McDonald ${ }^{1}$, Erkan Oterkus ${ }^{2}$ \\ ${ }^{1}$ Wind and Marine Energy Systems Centre for Doctoral Training, Electronic and Electrical \\ Engineering Department, University of Strathclyde, Royal College Building, 204 George St, Glasgow, \\ G1 1XW, UK \\ ${ }^{2}$ Naval Architecture and Marine Engineering Department, University of Strathclyde, 100 Montrose St, \\ Glasgow G4 0LZ, UK \\ pablo.jaen-sola@strath.ac.uk; alasdair.mcdonald@strath.ac.uk; erkan.oterkus@strath.ac.uk
}

\begin{abstract}
Direct-drive permanent magnet generators for multi-MW wind turbines are low speed high torque electrical machines requiring large, heavy and robust structures to maintain the airgap clearance open and stable. The structural mass of radial-flux generators can be estimated at an early phase of the design process. Using distinct approaches, the integrity of a $3 \mathrm{MW}$ electrical machine structure has been addressed from a dynamic perspective by carrying out modal analyses with a view to minimize its mass. A versatile tool has also been developed to help the engineers with the dynamic design of generator disc structures. Conical structures have been analysed and compared with the baseline disc model obtaining very promising results. Potential improvements have been proposed for more ambitious structural designs.
\end{abstract}

Keywords: Direct-Drive Wind Turbine; Generator Structure; Dynamic Design; Modal Analysis

\section{Introduction}

The recent concerns on global warming and the rapid increase in energy demand are taking governments and institutions to look at renewable energies as a potential solution. In this context, wind energy industry has a foremost role to play. Considered as the most consolidated among the renewable energy types, wind power has been widely researched and developed. Wind energy gathering devices have been deployed onshore and offshore. Due to the larger wind resources and steadier conditions taking place in deep waters as well as the fast development of the technology, offshore wind energy has recently drawn more attention.

However, significant challenges have to be considered when planning and installing offshore wind farms. The sea is a harsh environment which does not always allow an easy access to the turbines for installation or maintenance, where the air has a high salt content and humidity. In the installation case, both the wind speed and wave height must be below certain levels so that cranes and vessels can work safely. In addition, if a scheduled maintenance cannot be carried out because of extreme conditions, a very high cost produced by the downturn in the turbine availability will be paid by the owner. This is 
why manufacturing reliable elements at a minimum cost is of high importance. Lightweight and easy to install structures are of interest although not always possible since generators and other components such as gearboxes can be very complex and heavy.

Over the past years, the use of non-conventional drivetrain configurations for offshore purposes has arisen. With a view to increase the reliability and availability of the turbine, the direct-drive concept has been introduced. Directly driven wind turbines present a number of advantages, among which the removal of the gearbox stands out. With it expensive gearbox matters can be avoided. Moreover, the decrease in the number of moving parts represents a significant reduction in downtime periods. On the other hand, the rotational speed of large multi-MW wind turbines is very low (in the order of 10rpm) and knowing that the power of a generator is equal to the product of the machine's torque ' $T$ ' and the angular velocity ' $\mathrm{P}$ ', $(P=T \mathrm{P})$, very large torques must be developed by direct-drive machines. Practical limits to electrical and magnetic loading exist, thus there is an upper threshold to the shear stress that can be produced. A typical range for the shear stress, ' $\sigma$ ', goes from 25 to $50 \mathrm{kN} / \mathrm{m}^{2}$ [1]. Since the torque for an electrical generator can be calculated as

$$
T=2 \pi \sigma R^{2} l
$$

where $R$ corresponds to the radius of the machine's airgap and 1 is the axial length, a very large device of considerable mass should be considered.

Minimising the structural mass of low speed multi-MW electrical machines for renewable energy purposes have become an important object of study as with the reduction in mass a substantial decrease in the machine capital cost can be achieved [2]. Generators utilized in direct-drive wind turbines are all synchronous. One way of reducing its weight is moving from electrical excitation to permanent magnet excitation. Going a step beyond, permanent magnet machine's topology is also a variable that can be taken into account. There are three different types: radial-flux, axial-flux and transverse-flux and can be contrasted by looking at either their cost per torque or their torque density. Although axial-flux and transverse-flux machines are of substantial interest, this paper concentrates on radial-flux generators.

The vast majority of the research in the field focuses all the attention on the mass decrease of 'active material', which includes permanent magnets, copper and back iron. Nevertheless, it is the supporting structure, also known as 'inactive material', which accounts for the highest percentage of mass. The excessive weight of the generator structure was highlighted by Hartkopf et al.. who claimed that 2/3 of a direct-drive radial-flux electrical machine mass corresponded to the inactive material [3]. Mueller, McDonald and MacPherson claimed in [4] that at multi-MW ratings the inactive mass of a direct-drive axial flux machine is almost $90 \%$ of the total mass. A series of studies have been available on this regard presenting different approaches that can be utilised when designing these machines in order to minimize their structural mass [5][6]. In these investigations, analytical and numerical analysis techniques were used with the objective of finding the minimum required stiffness so that the machine can withstand the loads. As known, the structural stiffness, ' $k$ ', is the ratio between the force applied on a body, ' $F$ ', and the deflection produced by the force along the same degree of freedom, ' $\delta$ ', $(k=F / \delta)$. By having the 
minimum stiffness, the designers are able to estimate the minimum structural mass in a low cost and fast manner.

It is important to highlight the fact that all of the mentioned studies have adopted a static approach when analysing the machine. In this paper, the machine's structure has been studied from a dynamic point of view, where much deeper understanding is necessary. Only a few papers have looked in detail at this topic and most of them are centred on a particular machine. For instance, in [7], Sethuraman successfully characterised the dynamic behaviour of the direct drive power train of a spar-buoy floating wind turbine following the well-known two step de-coupled approach proposed by Xing et al. [8][9]. This method, originally created for analysing conventional geared wind turbines, utilises the data obtained from an aero-elastic simulation code, on global motion response and loads as inputs for a detailed drivetrain model created in a Multi-Body Simulation piece of software which enables kinematic and dynamic analyses of mechanical systems. However, as the main purpose of this research was to optimise a direct drive machine, the author also needed to address how significant the dynamic effects and feedback forces from the drivetrain were. In [10], Kirschneck employed density based topology optimisation to investigate the potential weight reduction of the XD-115 offshore direct-drive wind turbine generator rotor taking into consideration its dynamic behaviour. Here, the authors have developed a more generic approach that can be used for the excitation frequencies evaluation and alteration of any radial-flux electrical machine. As a rotating piece of machinery, the generator vibrates when its natural frequencies are excited introducing potentially large amplitude oscillations into the forces acting on it that could cause structural fatigue, noise and, in the worst case scenario, the sudden collapse of the structure.

Due to the special nature of its construction and operation, the way of evaluating the vibration of a wind turbine differs from that of any other industrial rotating machine. Vibrations of the tower and nacelle caused by the effect of wind, flow disturbances due to the tower, the natural vibration of the rotor blades and structure itself, and additionally sea swell, in the offshore case, are the sources of excitation to be considered [11]. Regarding the wind turbine components dynamics, few studies have been published on the design of large diameter direct-drive generator structures. Typical generator supporting structures are formed by disc, arm or conical sub-structures, which not only connect the shaft with the cylindrical sub-structure but also provide the necessary stiffness in the radial and axial directions. See Figure 1. Depending on the rated power and the requirements to comply with, the structure shape and dimensions will vary, as well as its natural frequencies and mode shapes. In this investigation, disc and conical structures have been studied. It was assumed that an elastic coupling, similar to Alstom's Pure Torque [12], has been placed between the wind turbine rotor and the electrical generator so that all the nontorque loads are directly diverted to the tower. With this and the appropriate bearing configuration, the electrical generator is virtually isolated from the rest of the turbine. In addition, an elastomeric-hydraulic coupling system has been assumed between the generator and the turbine turret that helps dissipate the vibrations induced by the sea swell. Taking all of these facts into account, the potential excitation frequencies that could activate the structure natural frequencies are as follows [13], 
The frequency of the wind turbine rotational speed (1P).

The fundamental electrical frequencies ( $p \mathrm{P}$, with $\mathrm{p}$ being the number of pole pairs).

The frequency of the rotor blades passing in front of the tower (3P and 6P).

Any mode shape, if sufficiently excited can lead to noise and fatigue damage. In order to explore the sufficient number of natural frequencies you may want to check as many modes as it takes to fully analyse the frequency excitation range you are expecting. This usually means that you need to look at 6 modes minimum and do not evaluate more than 10 modes or two hundred Hz. Nonetheless, one can adopt a more precise approach, where the energy contained within each resonant mode is measured so that the amount of system mass participating in each mode and for a particular direction can be estimated. The amount of mass is normalized and the retrieved value is known as the effective mass participation factor or EMPF [14][15]. A mode with large effective mass is typically a substantial contributor to the dynamic system response and must be avoided or passed as quickly as possible. If the sum of all EMPF corresponds to at least $80 \%$ in any response direction (X, Y, Z), it can be assumed that the number of modes studied is sufficient to capture the dominant response of the structure. Another important point that this procedure takes into account is that if one mode contributes more than $1 \%$ of the total mass it must be considered to avoid resonant issues [14][15][16].

The main aim of this investigation is to develop suitable methods and investigate a set of possible designs that would ensure the dynamic integrity of the machine without significantly increasing its weight by following the effective mass participation factor procedure described above.

\section{Methodology}

This study concentrates on electrical generator supporting structures formed by disc or conical substructures and the different methods that can be followed when approaching their dynamic design.

First, the potential excitation frequencies that can trigger the structure's natural frequencies are identified and assessed. Second, the natural frequencies of a statically optimised structure made with discs are estimated by using finite element techniques and plotted with the excitation frequencies on an interference diagram, also known as Campbell diagram, for comparison. This structure was considered the baseline model. The main objective of the static structural optimization was to minimise the structural mass while keeping the deflection within a certain specified limit corresponding to $0.5 \mathrm{~mm}$ in any direction [2]. For this analysis, the author constrained the structure at the shaft and introduced radial $(400 \mathrm{kPa})$, tangential $(30 \mathrm{kPa})$ and gravitational $\left(9.81 \mathrm{~m} / \mathrm{s}^{2}\right)$ loads. As said, it is crucial to elude or pass as quickly as possible any natural frequency with a large effective mass participation factor in order to avoid resonance. This gave us a clear picture of the generator range of operation and helped us find the most appropriate methods to maximize it. Those included the alteration of the thicknesses of the substructures, the use of different stiffeners and the change of structural shape (from disc to conical). 
A set of potential rotor and stator structures has been presented and described in [17]. Figure 1 displays some of the rotor structural models studied in other investigations,

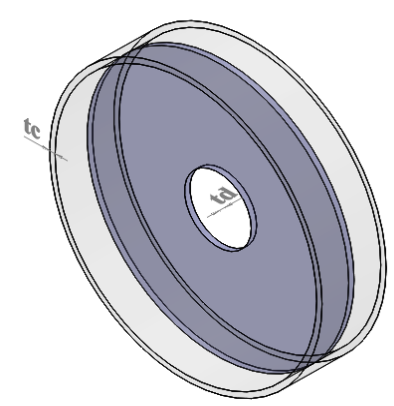

(a)

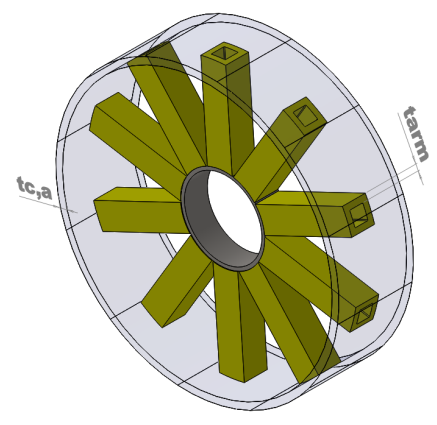

(b)

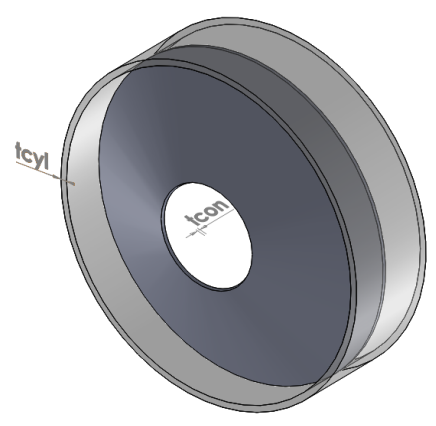

(c)

Figure 1 Rotor structures with thickness dimensions; (a) Disc structure; (b) Arm structure; (c) Conical structure [18]

where $t_{\mathrm{c}}$ and $t_{\mathrm{d}}$ are the cylinder and disc thicknesses of the structure made with discs, respectively; $t_{\mathrm{c}, \mathrm{a}}$ and $t_{\text {arm }}$ are the cylinder thickness and the hollow arm thickness of the armed structure, whereas, $t_{\mathrm{cyl}}$ and $t_{\text {con }}$ are the thicknesses of the cylinder and the cone of the conical structure. These are only support structures, also known as inactive material, and do not include back iron.

The disc or conical sub-structures not only connect the wind turbine rotor shaft to the generator rotor surface, but also provide the rotor and the stator with the necessary radial and axial stiffness to comply with the structural requirements demanded during the stages of assembly and transportation of the machine as well as during its operation. These two types of structural configurations have shown better performances under static loading than their armed (made with arms) counterparts [18], therefore they have been considered the ideal candidates for the dynamic studies.

Once the structural configurations showing the best performance under static loading conditions have been identified, linear dynamic analyses of the models have been carried out using finite element techniques so that their natural frequencies, also called resonant frequencies, could be found. The design information of the studied baseline machines has been listed in Table 1 for clarification.

Table 1 Baseline machine design characteristics

\section{Rated power}

Rated wind speed

Rated rotational speed

Cut in wind speed

Cut off wind speed

Airgap size
3MW

$11 \mathrm{~m} / \mathrm{s}$

9rpm

$3.5 \mathrm{~m} / \mathrm{s}$

$24 \mathrm{~m} / \mathrm{s}$

$5 \mathrm{~mm}$ 


$\begin{array}{ll}\text { Diameter } & 4 \mathrm{~m} \\ \text { Axial length } & 1.2 \mathrm{~m} \\ \text { Shaft diameter } & 0.625 \mathrm{~m} \\ \text { Aspect ratio } & 0.3 \\ \text { Structural configuration } & \text { Structure made with discs } \\ \text { Rotor dimensions } & t_{\mathrm{d}}=40 \mathrm{~mm} ; t_{\mathrm{c}}=37.5 \mathrm{~mm} \\ \text { Rotor structural mass } & 8,120 \mathrm{~kg} \\ \text { Stator dimensions } & t_{\mathrm{s}, \mathrm{d}}=20 \mathrm{~mm} ; t_{\mathrm{s}, \mathrm{c}}=33 \mathrm{~mm} \\ \text { Stator structural mass } & 9,451 \mathrm{~kg} \\ \text { Generator structural mass } & 17,571 \mathrm{~kg}\end{array}$

The piece of software selected to do the task was SolidWorks. This 2D/3D CAD modelling programme is a powerful tool that can also be used to run detailed finite element studies through a series of add-on packages, which include structural simulation, fluid dynamics and electrical analysis [19]. The steps given to run the frequency studies are described in detail in Section 2.1. The retrieved data and the potential excitation frequencies have been plotted on a Campbell diagram for comparison. With this, it has been possible to find the range of operation for these baseline models that help us determine the potential methods that can be utilized to alter the natural frequencies of the structures and adjust the range of operation according to the designer necessities. See Section 2.2.

Figure 2(a) illustrates a direct-drive wind turbine drivetrain model courtesy of Alstom/GE that can be simplified as a complete generator structure fully integrated within the wind turbine as it is shown in Figure 2(b).

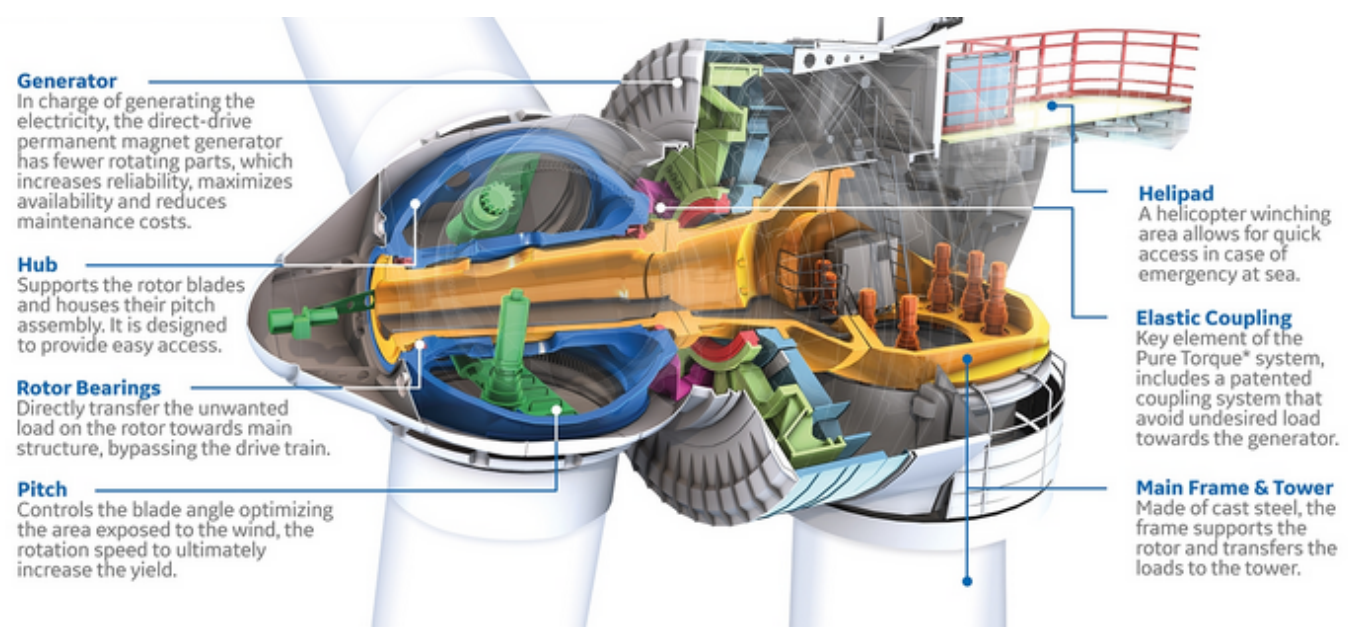

(a) 


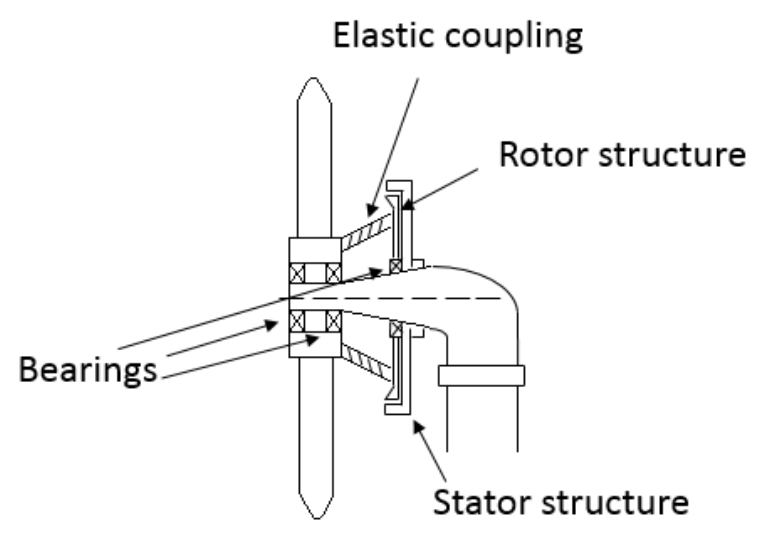

(b)

Figure 2 (a) Alstom/GE Haliade 150-6MW offshore direct-drive wind turbine [20]; (b) Fully integrated generator structure

As it can be observed, the Alstom/GE generator supporting structure is formed by a rotor structure made with a topology optimised disc, which has a series of stiffeners with different shapes attached to its axial surface as well as to the cylinder sub-structure providing radial and axial stiffness and conveniently altering its natural frequencies. The generator bearing inner race is attached onto a spindle connected to the stator frame while the outer race is connected to the generator rotor. The stator support frame is anchored to the turret and carries the stator structure. It is made up with a topology optimised disc structure having elastomer-hydraulic couplings, as shown in Figure 3, to dissipate the tower vibrations to be transmitted to the electrical generator as the movement of the liquid gives the possibility of introducing additional damping into the system. The bearing configuration and the elastic coupling (Alstom's Pure Torque) located between the turbine rotor and the generator protects the electrical machine from misalignments and makes it more compact.

In this paper, a similar bearing wind turbine configuration with an inner generator rotor has been assumed for the study of all support structures. With the aim of completely isolating the generator from the rest of the turbine an elastic coupling, similar to Alstom's Pure Torque [12], has been also considered to be located between the wind turbine rotor and the electrical machine so that all the non-torque loads are directly transmitted to the tower. Moreover, a damped connection of the generator to the turret by means of elastomer-hydraulic couplings ensures that no stresses are produced in the mechanical drivetrain in the case of structural deflections, prevents the transmission of structure-borne noise and helps dissipate the vibrations induced by the sea swell. Figure 3 shows the system assumed. 


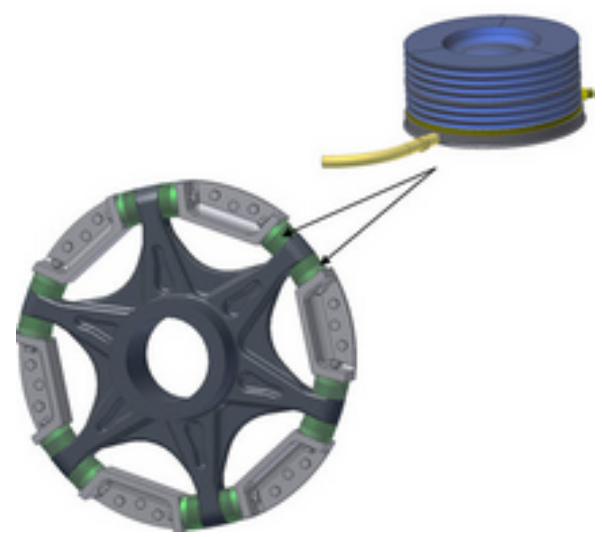

Figure 3 Elastomer-hydraulic coupling installed on the stator frame for the generator and turret connection [21]

As presented, several investigations have been carried out with the main aim of minimizing the mass of the electrical machine while maintaining its structural integrity. However, all of them analysed the structure from a static viewpoint. With very large torques and very low rotational speeds, it is vital to find distinct and innovative ways of altering the natural frequencies of the supporting structures in order to avoid resonance while maximizing generator operating range. A deeper understanding is needed if the minimization of the structural mass is also a mandatory requirement. Having that in mind, different approaches have been explored and new tools, which can be used during the early phases of the design, have been introduced. The bearings stiffness has not been considered in any of the studies.

\subsection{Evaluating structural natural frequencies}

A finite element modelling package, such as SolidWorks, was employed so as to estimate the lowest mode shapes of the generator structures. The theory behind the modal analyses carried out with this piece of software leans on the principle described in [22]. With a modal analysis one can study the dynamic characteristics of a structure in the frequency domain. It uses the structural stiffness and mass to encounter the periods at which the structure will naturally resonate. The evaluation of resonant frequencies of very simple structures having only one degree of freedom can be done by using Equation 2

$$
f=\frac{1}{2 \pi} \sqrt{\frac{k}{m}} .
$$

where, $f$ is the natural frequency, $k$ is the stiffness and $m$ corresponds to the mass. However, this evaluation would require the use of more complex equations or stress analysis packages depending on the complexity of the structural configuration, which is the case of rotor and stator structures. Having said that, it is important to highlight the fact that it is possible to avoid resonance by influencing the structural stiffness or mass or both. 
In [6], the authors found through comparison that generator disc structures are much lighter than armed structures working under same loading conditions $(400 \mathrm{kPa}$ radial load, $30 \mathrm{kPa}$ tangential load and gravity conditions of $9.81 \mathrm{~m} / \mathrm{s}^{2}$ ) and constraints (structure constrained at the shaft) as the hollow arms cannot cope with the tangential loading as well as the disc sub-structures do unless the thickness of the arms is considerably increased with the subsequent rise in mass. With the optimum configuration identified, the first 20 natural frequencies of the rotor and stator structures of a $3 \mathrm{MW}$ machine of $4 \mathrm{~m}$ diameter and $1.2 \mathrm{~m}$ of axial length have been addressed, by performing modal analyses. The modes giving more information about the dynamic response of the system were plotted in a Campbell diagram for comparison.

Figure 4(a)(b) displays the disc structural models utilized for the modal analyses showing constraints.

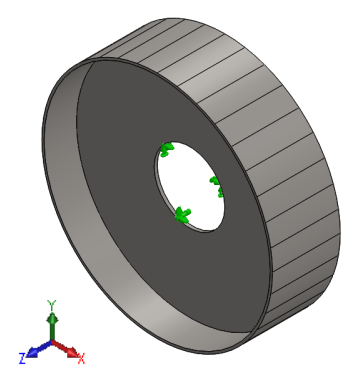

(a)

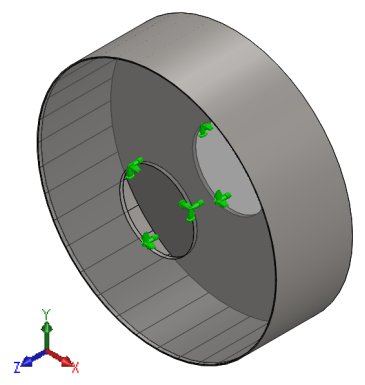

(b)

Figure 4 (a) Rotor structure showing constraints; (b) Stator structure showing constraints [18]

For that, the SolidWorks Simulation $\odot$ add-on package was used. The frequency analysis study option was selected among the distinct types of studies available. Then, structural steel was assigned to all components forming the structures as their material: Young's modulus, $E=2.1 \times 10^{11} \mathrm{~Pa}$, Poisson's ratio, $v=0.3$ and density, $\rho=8,000 \mathrm{~kg} / \mathrm{m}^{3}$. The next step was to constrain the structures at the shaft and create an appropriate mesh. In SolidWorks, it is possible to adjust the mesh element size manually by making use of a slider which goes from a finer mesh to a coarser one. The piece of software also suggests an element size by default that is supposed to give accurate results in a reasonable period of time. In addition, two types of adaptive meshes are available too: h-type and p-type. The h-adaptive mesh diminishes the size of the elements in an iterative manner, locally or globally depending on the settings specified by the user until convergence is accomplished, while the p-adaptive mesh increases the element order (linear, quadratic, cubic, etc,.) again locally or globally depending on the specifications in an iterative way until convergence is achieved. See Figure 5 for further clarifications on element order. In this case, similar results should be obtained by using any of these two techniques. 

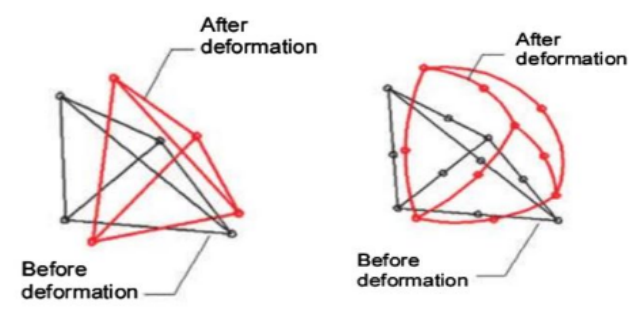

Figure 5 (a) First order (linear tetrahedral solid element with no nodes at the midpoints); (b) Second order for a higher quality mesh (parabolic tetrahedral solid element with nodes at the midpoints) [23]

For these studies the mesh density suggested by the FE package was utilised. A linear tetrahedral high quality mesh with 7,682 elements and 15,640 nodes was created. The element size was $148.5 \mathrm{~mm}$.

The estimation of the first twenty mode shapes of the rotor and the stator structures was performed with no loading applied at any time. This means that any damping or stiffness effects that may occur due to electromagnetic phenomena are neglected.

\subsection{Dynamically designing a direct-drive generator supporting structure}

Only a few studies have been published on the design of large diameter direct-drive generator structures. Most of them concentrate all the attention on the effects of the external loads acting on the whole drivetrain but very few look at how these forces affect the components forming the electrical machine structure individually. The potential excitation frequencies that could trigger the structure natural frequencies have been presented and defined in Section 1. As mentioned the most dangerous structure's natural frequencies must be avoided or passed as quickly as possible and can be influenced by modifying the structural stiffness or mass or both. If a component is weaker in one direction, in other words, the moment of inertia in that direction is the lowest, it exposes itself as a low frequency resonant mode. In this regard, designers of this type of devices often follow an empirical rule that most dangerous natural frequencies between $70 \%$ and $130 \%$ of the exciting frequency must be avoided [24]. The techniques described and analysed in this investigation, include the variation of the parts thicknesses, the implementation of distinct types of stiffeners and the use of alternative structural shapes. All of them help the designer adjust the structure's natural frequencies to a greater or lesser extent.

The lowest natural frequencies of both the rotor and the stator structures can be estimated utilising FE techniques. Different excitation frequencies are able to trigger the cited natural frequencies. This study has been carried out considering the three types of frequencies defined in the introduction section. With this, different options were explored so as to alter the natural frequencies of the structures without adding significant weight.

Considering a number of pole pairs equal to 60 , the excitation frequencies for the drivetrain were encountered for a wide range of rotational speeds going from 0 to $20 \mathrm{rpm}$. The outcomes were plotted in the frequency interference diagram shown in Figure 6 for comparison. 


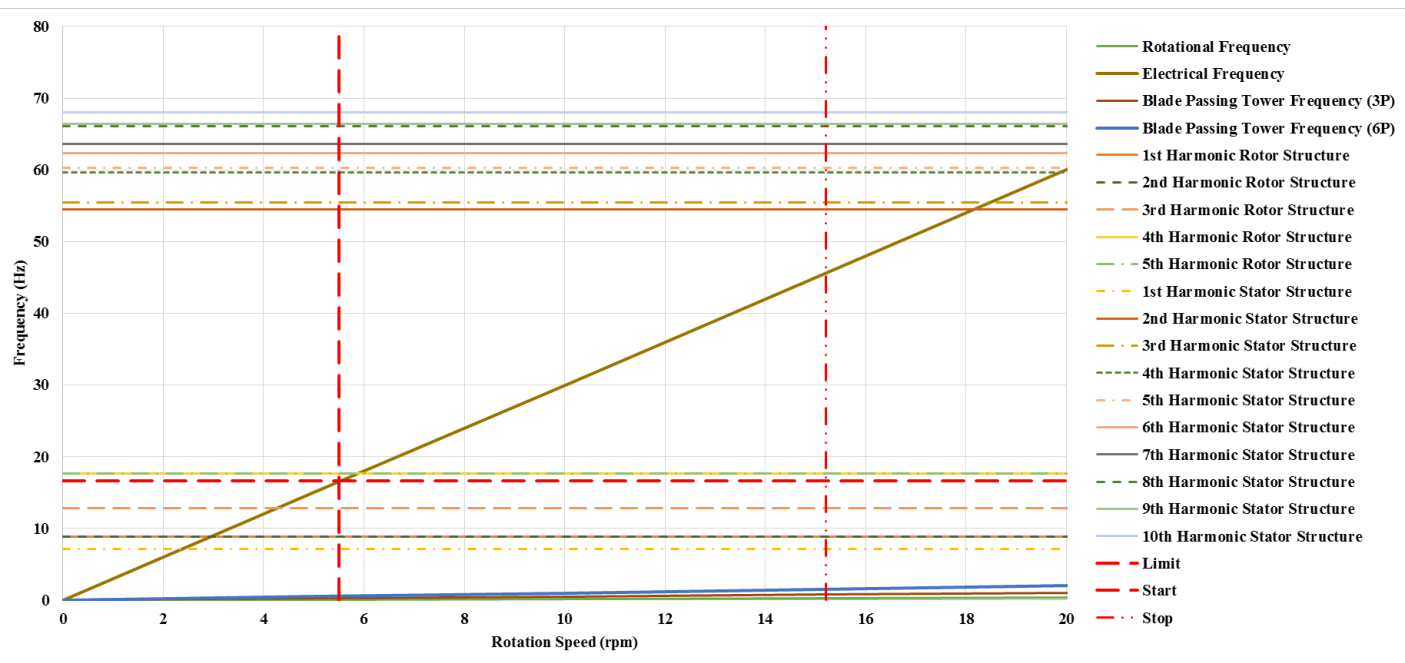

Figure 6 Campbell diagram of the system

An interference diagram, also known as Campbell diagram, named after Wilfred Campbell who first introduced the concept, represents the frequency in Hertz versus the rotational speed of the rotating piece of machinery in rpm, in this case the turbine rotor. The development of the natural frequencies corresponding to the mode shapes are presented as a function of the rotational speed of the rotor as horizontal lines. To excite the structure, any of the excitation frequencies, which in this graph corresponds to the inclined lines, have to intercept any of the natural frequencies having large effective mass participation factors (typically over $1 \%$ ).

Looking at the Campbell diagram, it can be seen that the weakest component is the stator with a frequency for the first mode of $7.1 \mathrm{~Hz}$, whereas the frequency of the first mode for the rotor is $8.8 \mathrm{~Hz}$. Nevertheless, as it was explained in Section 1, the dangerous mode shapes are those showing large effective mass in any direction. Moreover, it has been stated that if the sum of all the effective mass participation factors in any direction exceeds $80 \%$ of the total mass it can be considered that the number of modes studied is sufficient to capture the dominant dynamic response of the structure. Also, if one mode contributes more than $1 \%$ of the total mass it must be considered to avoid resonant problems. Figure 7(a) illustrates the effective mass participation factor for the rotor structure against the mode shape (frequency in $\mathrm{Hz}$ ). As seen, mode $3(13 \mathrm{~Hz}$ ) considerably exceeds the stipulated $80 \%$ in the $\mathrm{Z}$ direction. Among the rest of the analysed modes, only mode 12 goes beyond $1 \%$ of the total mass, again in the axial direction. Because of this, it can be said that mode 3 is the most dangerous mode for the structure and must be avoided, whereas as mode 12 , corresponding to $111.8 \mathrm{~Hz}$, is already above the $130 \%$ of the exciting frequency (electrical frequency) can be neglected. The total number of modes analysed is 20 so that the whole spectrum of generator operation can be explored with an additional tolerance. Figure 7(b) represents the cumulative effective mass participation factor where it can be seen that the $\mathrm{Z}$-axis direction is the weakest and that deviations take place at modes 3 and 12 . 


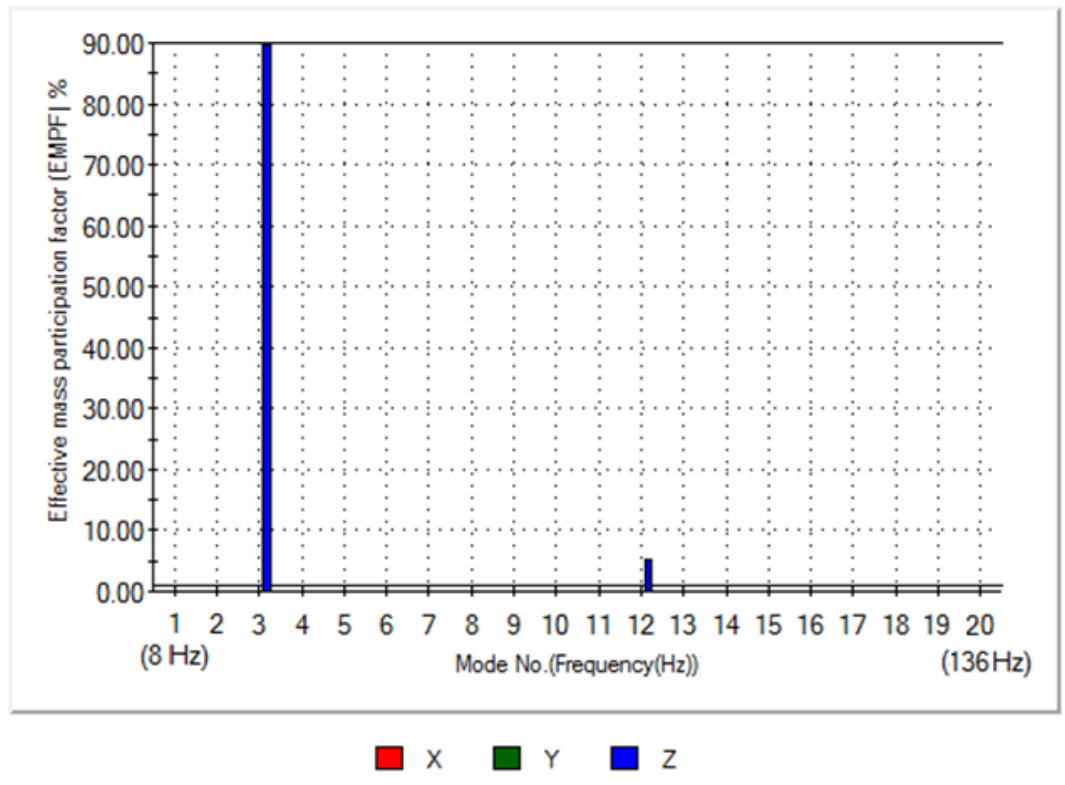

(a)

\section{Frequency vs.Cumulative effective mass participation factor (CEMPF)}

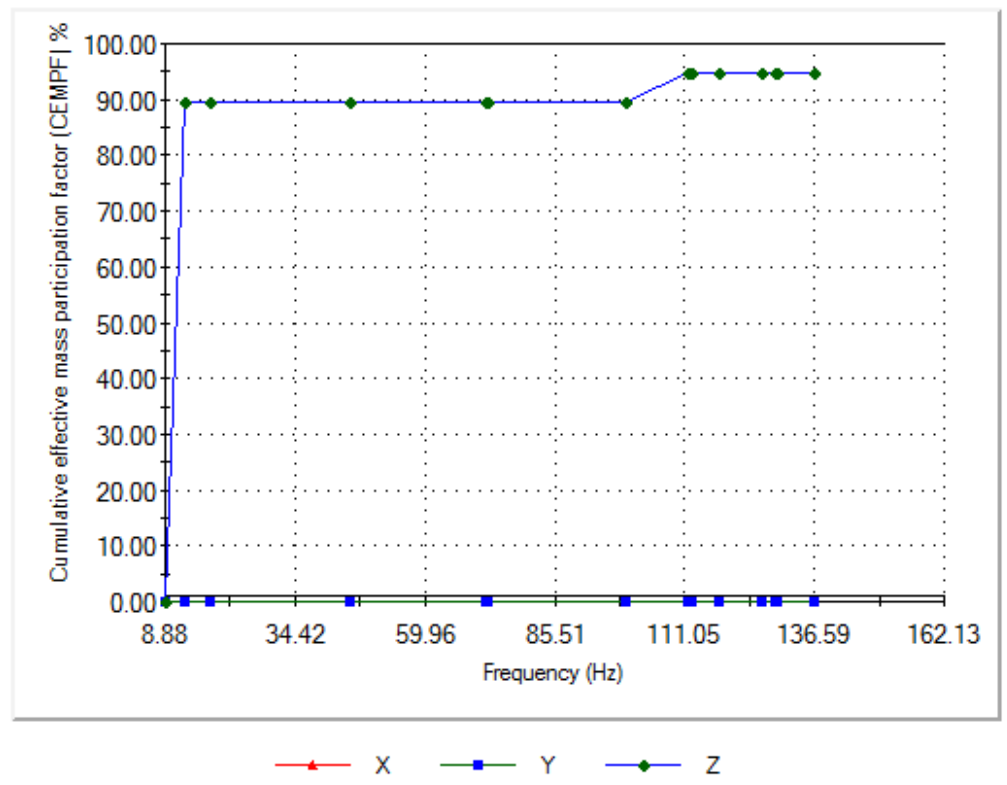

(b)

Figure 7 (a) Effective mass participation factor vs. mode shape ( Rotor structure); (b) Cumulative effective mass participation factor vs. mode shape (Rotor structure)

Figure 8(a) represents the effective mass participation factor of the stator structure against its natural frequencies. It can be observed how the effective mass participating in mode 1 represents more than $80 \%$ of the total mass again in the Z-axis direction. Some effective mass participates in modes 7,8 and 10 in the $\mathrm{Z}$ direction. As mode 8 does not go over the specified limit of $1 \%$, it can be said that it does 
not represent a risk to the structure, whereas modes 7 and 10 must be taken into consideration. Again 20 modes have been studied in order to cover the whole machine spectrum of operation with an extra tolerance. Figure $8(\mathrm{~b})$ shows the cumulative effective mass participation factor against the natural frequencies. Again, it can be observed that the moment of inertia in the axial direction ( $\mathrm{Z}$ axis) is the lowest and that the deviations happen at mode $1,7,8$ and 10 .

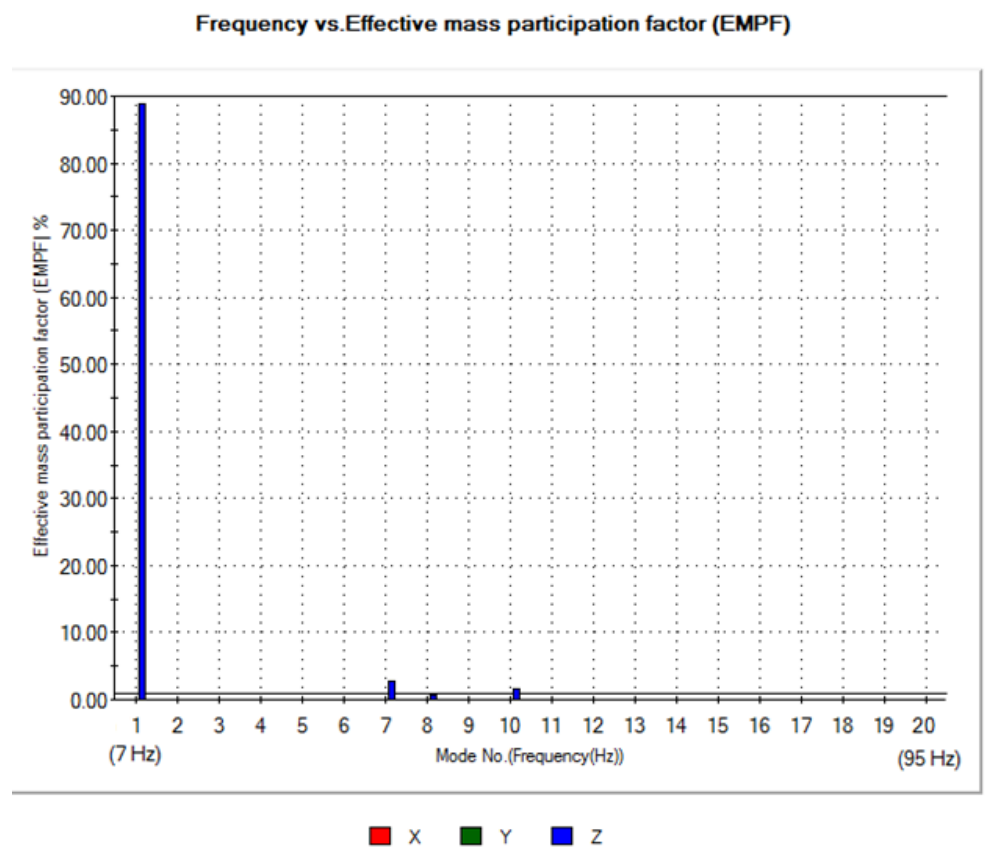

(a)

\section{Frequency vs.Cumulative effective mass participation factor (CEMPF)}

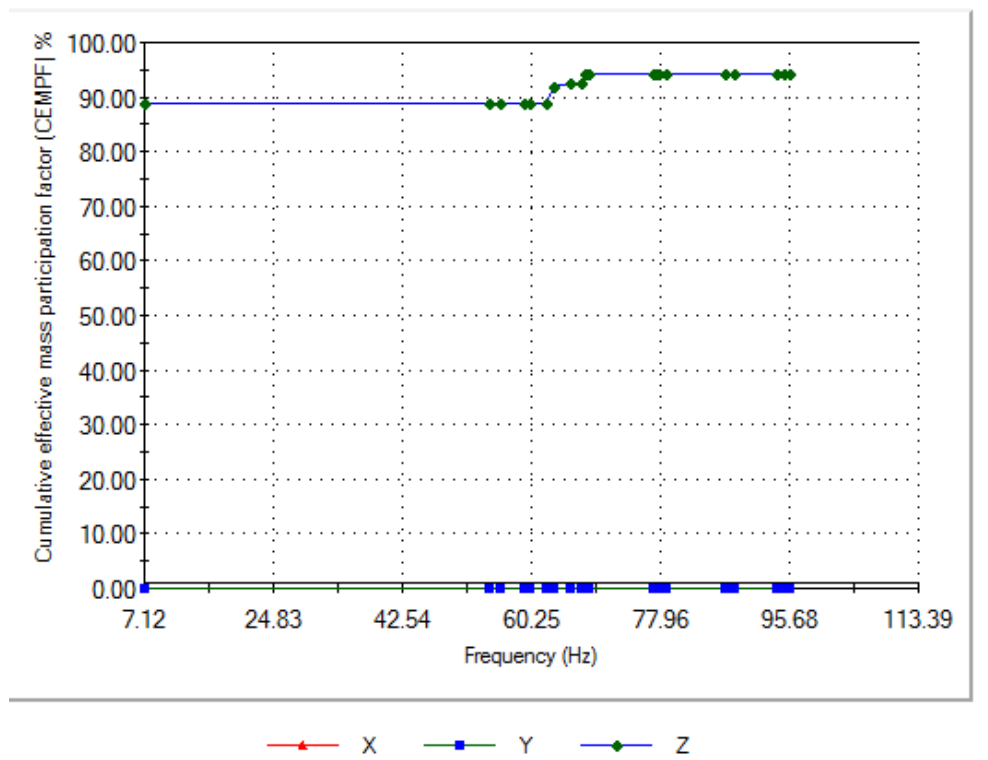

(b)

Figure 8 (a) Effective mass participation factor vs. mode shape ( Stator structure); (b) Cumulative effective mass participation factor vs. mode shape (Stator structure) 
As mentioned, the exciting frequency must be either above or below the critical natural frequency by $30 \%$. Then, by looking at the Campbell diagram (Figure 6) it can be understood that the $3^{\text {rd }}$ mode of the rotor structure and the $7^{\text {th }}$ mode of the stator structure define the operation range. Taking that into account, if one adds $30 \%$ to the $3^{\text {rd }}$ natural frequency of the rotor a frequency of $17 \mathrm{~Hz}$ is acquired (Limit line in the Campbell diagram), which cuts the inclined line corresponding to the electrical frequency at 5.5rpm (Start line in the Campbell diagram). Similarly, if one subtracts $30 \%$ to the 7 th mode of the stator, the frequency obtained is $44.5 \mathrm{~Hz}$, which corresponds to $15.2 \mathrm{rpm}$ (Stop line in the interference diagram). As a result, it can be assumed from Figure 6 that for a 3MW direct drive machine optimised structure made with discs the suitable range of operation is between 5.5 and $15.2 \mathrm{rpm}$. The need to avoid the lowest natural frequencies forces the designer to sacrifice the collection of energy at the lowest and the highest wind speeds for the good of the structure. Different ways of modifying the natural frequencies of the disc structures have been analysed in this paper. The results acquired for these tests are illustrated in detail in Section 3.

\subsection{Techniques for changing structure's natural frequencies}

There exist 4 distinct ways of changing the natural frequency of a design [25]:

- Alter the geometry.

- Strategically locate mass elements.

- Change materials (resonant frequencies are directly proportional to the elastic modulus of the materials)

- Modify the features of the shock isolators.

The first three ways have been looked at in this investigation. Since the structural design of the electrical machine has been approached from a general perspective, the fourth manner of varying the frequencies has not been studied.

Looking at the structural mode shapes obtained from modal analyses, the sort of technique that might apply better to change the natural frequencies can be identified.

\subsubsection{Dimensional alteration of structures made with discs}

As stated, a 3MW machine of $4 \mathrm{~m}$ diameter and $1.2 \mathrm{~m}$ axial length made up of steel, as shown in Figure 9, has been assumed. Dimensions $t_{\mathrm{c}}$ and $t_{\mathrm{d}}$ for the rotor, and $t_{\mathrm{cs}}$ and $t_{\mathrm{ds}}$ for the stator have been varied and modal analyses carried out so that the effect caused by those alterations in the natural frequencies could be studied and quantified. 

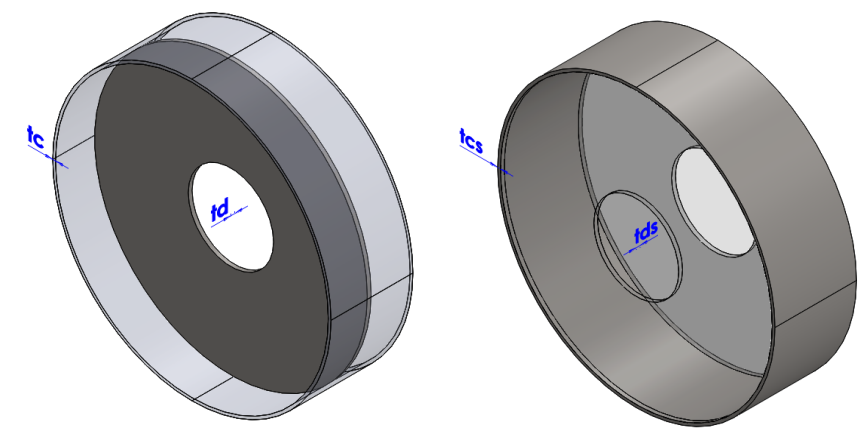

Figure 9 Rotor (left) and stator (right) structures as changed in the analysis

The thicknesses, measured in millimetres, of both rotor sub-structures were given the following values,

- Cylinder thickness, ' $t_{\mathrm{c}}$ ': 20, 25, 30, 35, 40 and $45 \mathrm{~mm}$

- $\quad$ Disc thickness, ' $t_{\mathrm{d}}$ ': $30,35,40,45,50,55,60,65,70,75$ and $80 \mathrm{~mm}$

whereas for the stator sub-structures the values given were,

- Cylinder thickness, ' $t_{\mathrm{cs}}$ ': 20, 25, 30, 35, 40, 45, 50 and $55 \mathrm{~mm}$

- $\quad$ Disc thickness, ' $t_{\mathrm{ds}}$ ': $10,20,30,40,50,60,70$ and $80 \mathrm{~mm}$.

These dimensions were chosen based on the values presented by Jaen-Sola in [6], in which contour plots are utilised to optimise the generator structure with a view to minimise its mass. In [6], contour plots display the stiffness and mass of the structures for different sub-structures thicknesses. With the disc thickness in the Y-axis and the cylinder thickness in the horizontal axis the lines plotted represent stiffness and structural mass. If the minimum stiffness necessary to comply with the structural requirements is known (structural deflection not allowed to exceed $0.5 \mathrm{~mm}$ or $10 \%$ of the airgap size in any direction), it is possible to trade off the mass between rotor and stator structures in order to find the lightest arrangement. In this case, contour plots display natural frequencies and structural mass and data used are all within a realistic range, which varies around the optimum results obtained from the static analyses carried out in [6]. A total number of 49 modal cases were analysed. The outcomes achieved were plotted and given in Section 3.1.

\subsubsection{Use of stiffeners}

Another way of manipulating the natural frequencies of the structures is through the introduction of other structural components, named stiffeners, which provide the structure with the necessary directional stiffness. When finding the structure's natural frequencies, special attention must be given to the mode shapes. The different forms adopted by the structure give the designer a clear picture of the type of stiffener required and their location. With this in mind, it is important to remember that the main aim of this investigation is to reduce the mass of the machine while meeting structural requirements. 
Therefore, the utilisation of stiffeners will be suitable only when a substantial alteration in frequency is observed without adding too much mass.

In this research, an isosceles triangular type of stiffener with a thickness of $60 \mathrm{~mm}$ joining the cylinder and the disc sub-structures has been proposed. The use of rectangular ribs of length $=1,200 \mathrm{~mm}$, width $=150 \mathrm{~mm}$ and thickness $=150 \mathrm{~mm}$ attached to the disc sub-structure was also suggested. Although these dimensions were randomly picked, it was intended to find suitable sizes that help us alter the frequencies in a significant manner. By looking at the mode shapes acquired from the baseline model analyses, it was possible to determine a suitable type of stiffener. With high shear stresses, which try to separate one sub-structure from the other, and the highest deflections taking place along the rim and on the outer edge of the disc sub-structure due to the disc sub-structure warping, a triangular type of stiffener joining both sub-structures and rectangular ribs placed on the disc sub-structure were considered good options to adopt. Besides its low manufacturing cost, triangular stiffeners have excellent shear stress resistance and are widely utilised in the construction industry [26]. Located in the axial direction, its equal length sides are joined to the inner face of the rim and the axial surface of the disc and its dimensions vary with the disc thickness. As shown in Figure 10(a), a finite number of stiffeners equally spaced have been employed. First on one side of the disc only and then on both sides. Ribs stiffeners are extensively used in the construction industry as well due to their low cost of manufacturing and versatility and in this study have been placed on the disc sub-structure of both rotor and stator with the aim of eluding the warping. In Figure 10(b), it can be seen how for the rotor they were first attached to one side and then to both. For the stator, they were placed on the outer side of one disc first and second on both outer surfaces of both discs. See Figure 10(c). Triangular stiffeners were not tested on stator structures and rib stiffeners were not analysed on the inner faces of the stator disc sub-structures as they would force the designer to modify the entire generator assembly so as to avoid physical collision between rotor and stator.

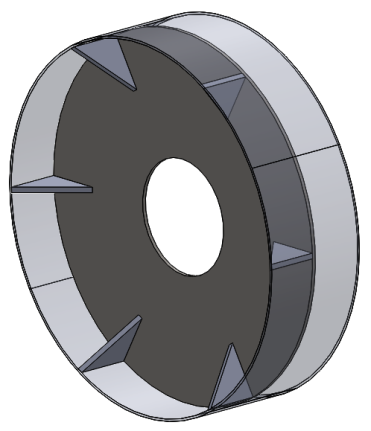

(a)

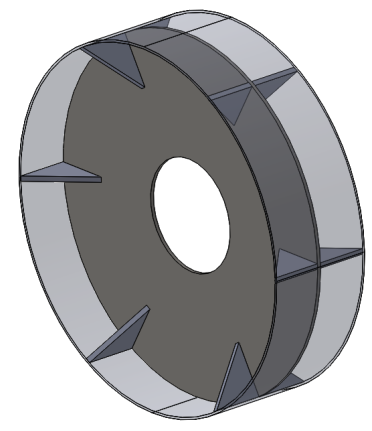

(b) 


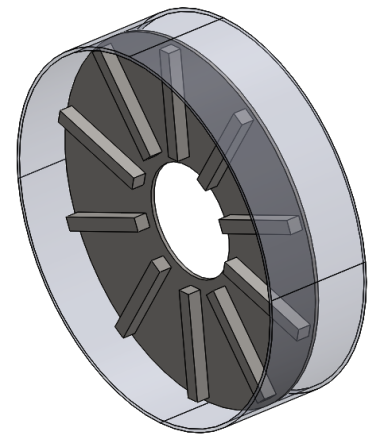

(c)

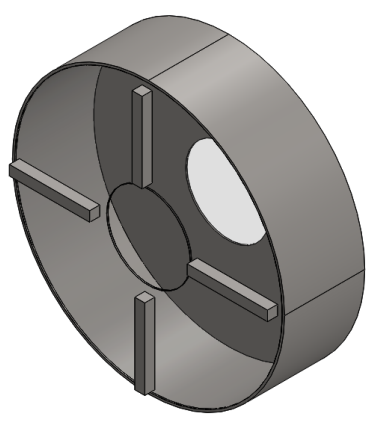

(e)

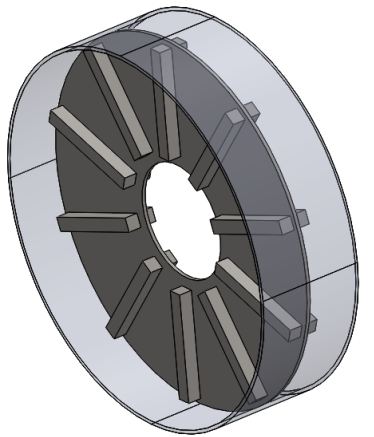

(d)

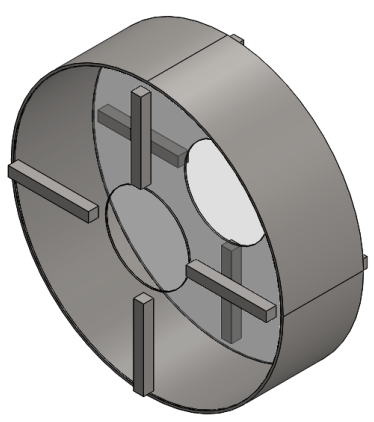

(f)

Figure 10 Rotor structures with axial stiffeners; (a) Triangular stiffeners on one side of the rotor; (b) Triangular stiffeners on both sides of the rotor; (c) Rib stiffeners on one side of the rotor; (d) Rib stiffeners on both sides of the rotor; (e) Rib stiffeners on one side of one of the stator discs; (f) Rib stiffeners on one side of both stator discs;

A different number of stiffeners as well as the use of a distinct material with lower density (alloy steel) in their design were also tried with the main aim of minimising the overall mass. Again, all the outcomes obtained from this part of the investigation are given in Section 3.2.

\subsubsection{Rotor conical structure}

In the last sub-sections, the techniques utilised for incrementing the natural frequencies of an optimised disc structure have been presented. However, other types of simplified structural configurations exist as stated by Stander in [17].

Rotor conical structures provide an axial stiffness that a disc structure misses, while the cone substructure acts as a disc sub-structure bringing radial stiffness to the model. A rotor conical structure has been optimised according to the deflection criterion as applied to the disc structure (deflection cannot exceed $0.5 \mathrm{~mm}$ in any direction) and under the same loading conditions as described in [18], which implies a radial load of $400 \mathrm{kPa}$ and a tangential load of $30 \mathrm{kPa}$, as well as considering gravity conditions of $9.81 \mathrm{~m} / \mathrm{s}^{2}$, but also taking into account its natural frequencies. Once again, the use of contour plots 
has been proposed for this task. The thicknesses, measured in millimetres, of both rotor sub-structures were given the following values,

- Cylinder thickness, ' $t_{\text {cyl }}$ ': 20, 25, 30, 35, 40 and $45 \mathrm{~mm}$

- $\quad$ Cone thickness, ' $t_{\text {con }}$ ': $10,20,30,40,50$ and $60 \mathrm{~mm}$.

The optimisation of this type of structure presents more complexity than others as more variables, such as cone sub-structure position and cone angle, ' $\psi$ ', need to be introduced into the model. The acquired results are given in Section 3.3.

\section{Results}

As explained in Section 2, the natural frequencies having large EMPFs of the generator structure must be eluded in order to avoid resonance. Different techniques can be used to alter natural frequencies. In this section, the data retrieved from the analyses carried out over 3 distinct types of structural configurations are presented.

At this point, it is important to mention that the rotor and the stator structures have been studied individually and the potential interactions between them have not been taken into consideration. An inner rotor type was utilised in the studies. The results shown in this section do not apply to machines with outer rotor configurations as the change in geometry will produce different outcomes for the same loading conditions.

Figure 11 displays the shapes of the third mode of the baseline rotor disc structure model and the shapes for mode 1,7 and 10 of the stator. The excitation of the third natural frequency of the rotor and the first of the stator would produce a lateral deflection in the $\mathrm{Z}$ direction of both the cylinder and disc substructures. As the disc is fixed at the shaft, the deflection in the $\mathrm{Z}$ direction would induce the disc substructure to acquire a conical shape. In the last two modes presented here (stator modes 7 and 10), the disc sub-structures show large deflection at its axial surface taking place periodically that produce bumps. Although these modes seem to produce random shapes, it is not difficult to recognise a deflection pattern. The largest deflection is located on the disc and it follows a circular shape that expands around the $\mathrm{Z}$ axis and eventually splits up into other smaller bumps as it can be appreciated.

These modes obtained due to the excitation of the structure's natural frequencies create an uneven deflection across the structure that would introduce such instability in the airgap that could cause the collapse of the machine. Deflections given in scale are in $\mathrm{mm}$ and are only for reference. Two options can be taken into account to skip this situation:

- To make use of the techniques given in Section 2.

- To design a structure capable of withstanding the stress without significantly deforming.

Note that the latter would imply a substantial increase in the structural mass. 


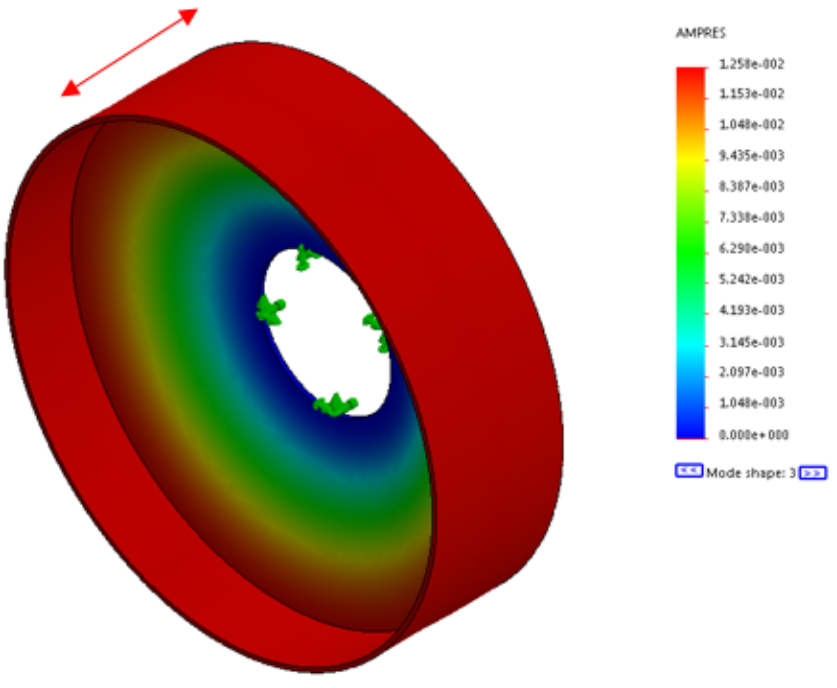

(a)

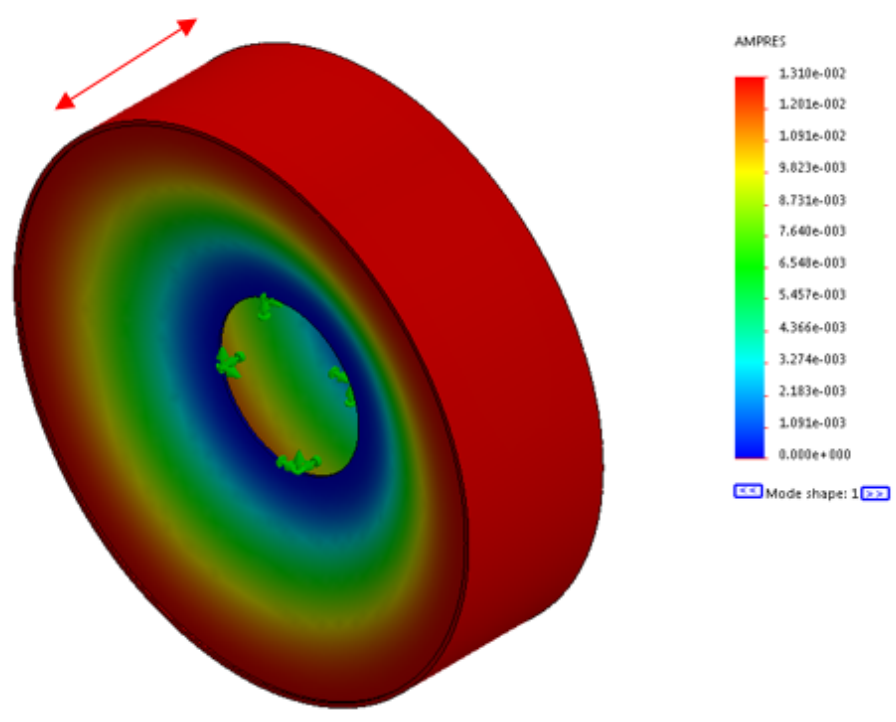

(b) 


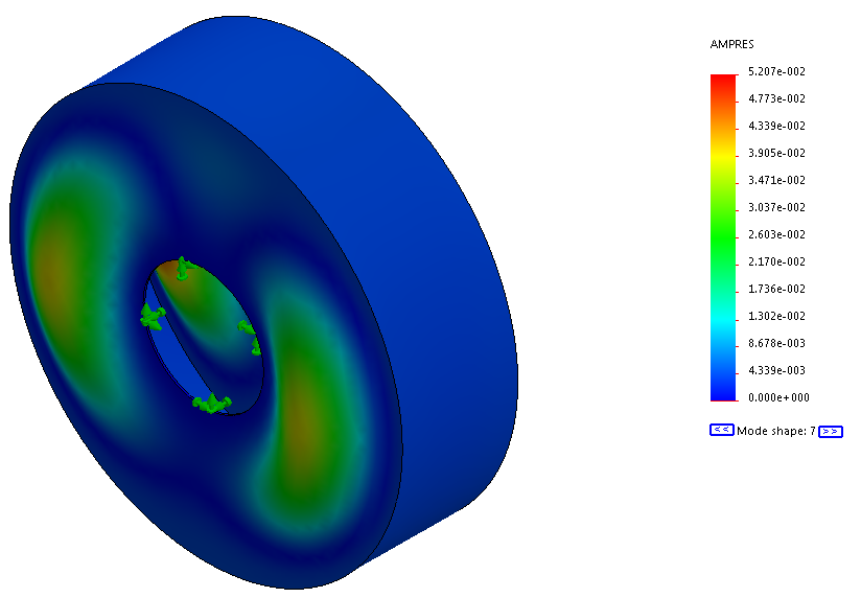

(c)

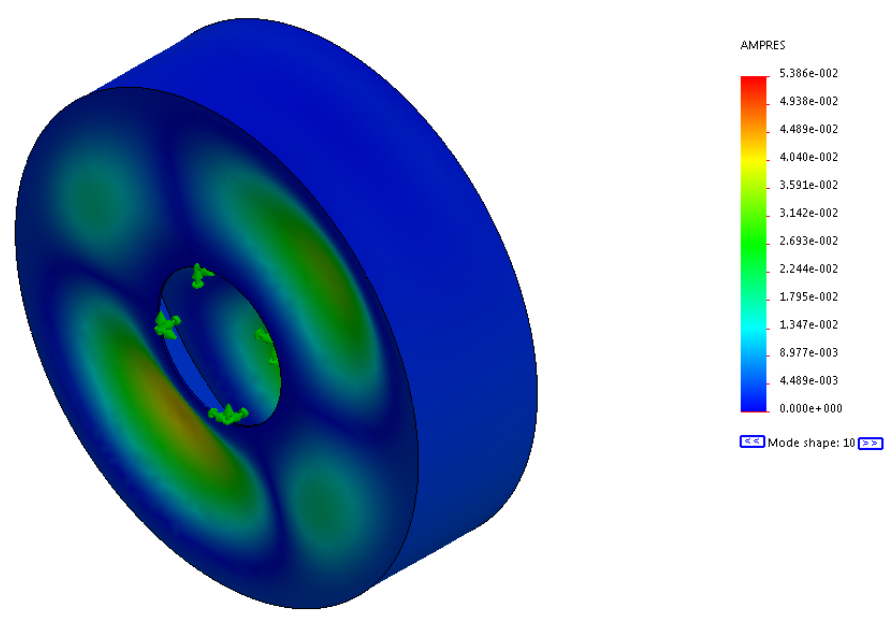

(d)

Figure 11 Rotor and stator disc structure modes; (a) $3^{\text {rd }}$ rotor mode $=13 \mathrm{~Hz}$; (b) $1^{\text {st }}$ stator mode $=7.13 \mathrm{~Hz}$; (c) $7^{\text {th }}$ stator mode $=63.64 \mathrm{~Hz}$; (d) $10^{\text {th }}$ stator mode $=67.96 \mathrm{~Hz}$

\subsection{Disc structure optimisation using contour plots}

Once the most dangerous natural frequencies of the baseline structure model have been identified, its structural mass optimisation can be carried out using contour plots. One can plot the mass of a disc structure on a $2 \mathrm{D}$ plot, with cylinder thicknesses on the X-axis, disc thicknesses on the Y-axis, and contours representing rotor or stator structural mass. Another set of contours can be used to show the natural frequency. See Figure 12. The frequencies presented in these plots correspond to the third mode shape of the rotor structure and to the first, seventh and tenth mode shapes of the stator structure, respectively. It was observed that the alteration of sub-structure thicknesses do not change the modes 
showing the dominant dynamic response. Therefore, the use of more contour plots was considered unnecessary.

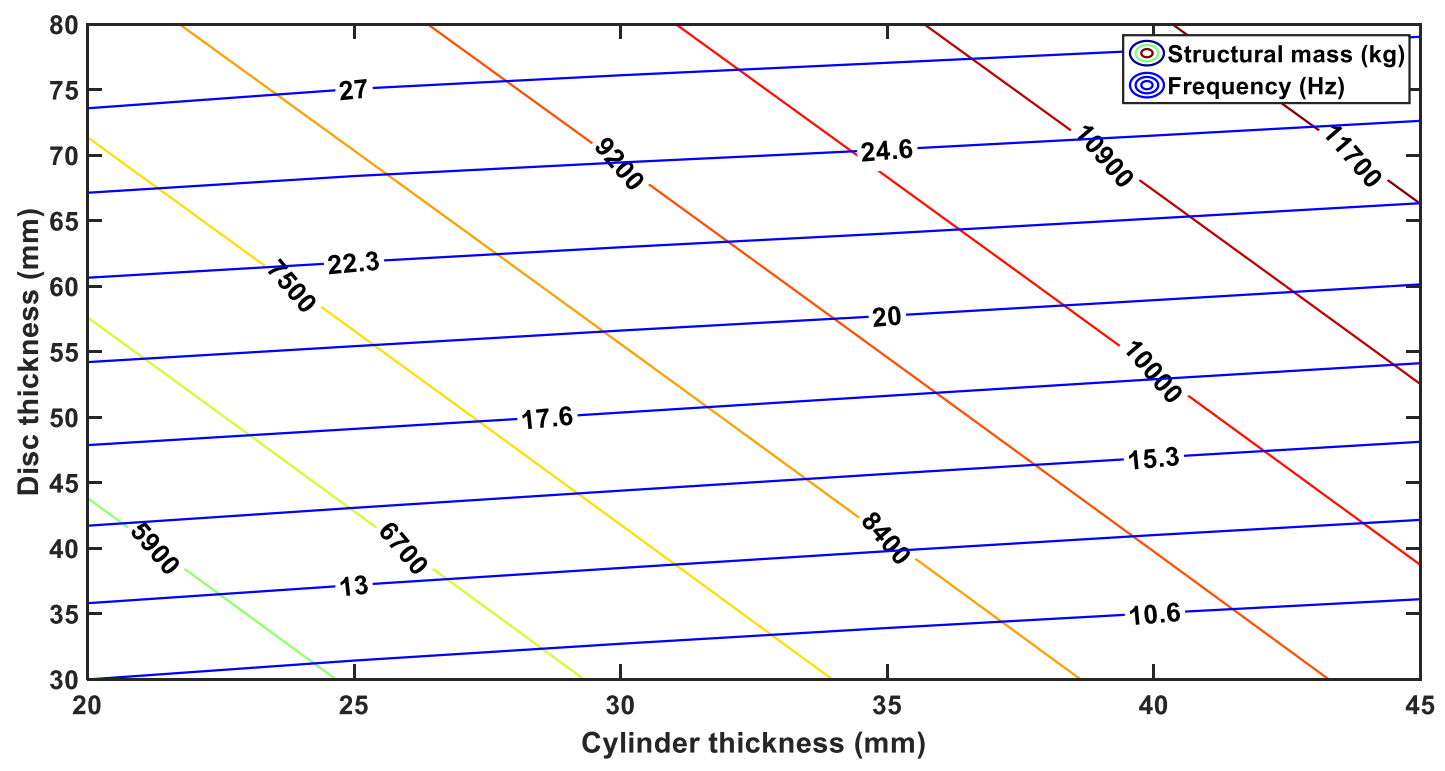

(a)

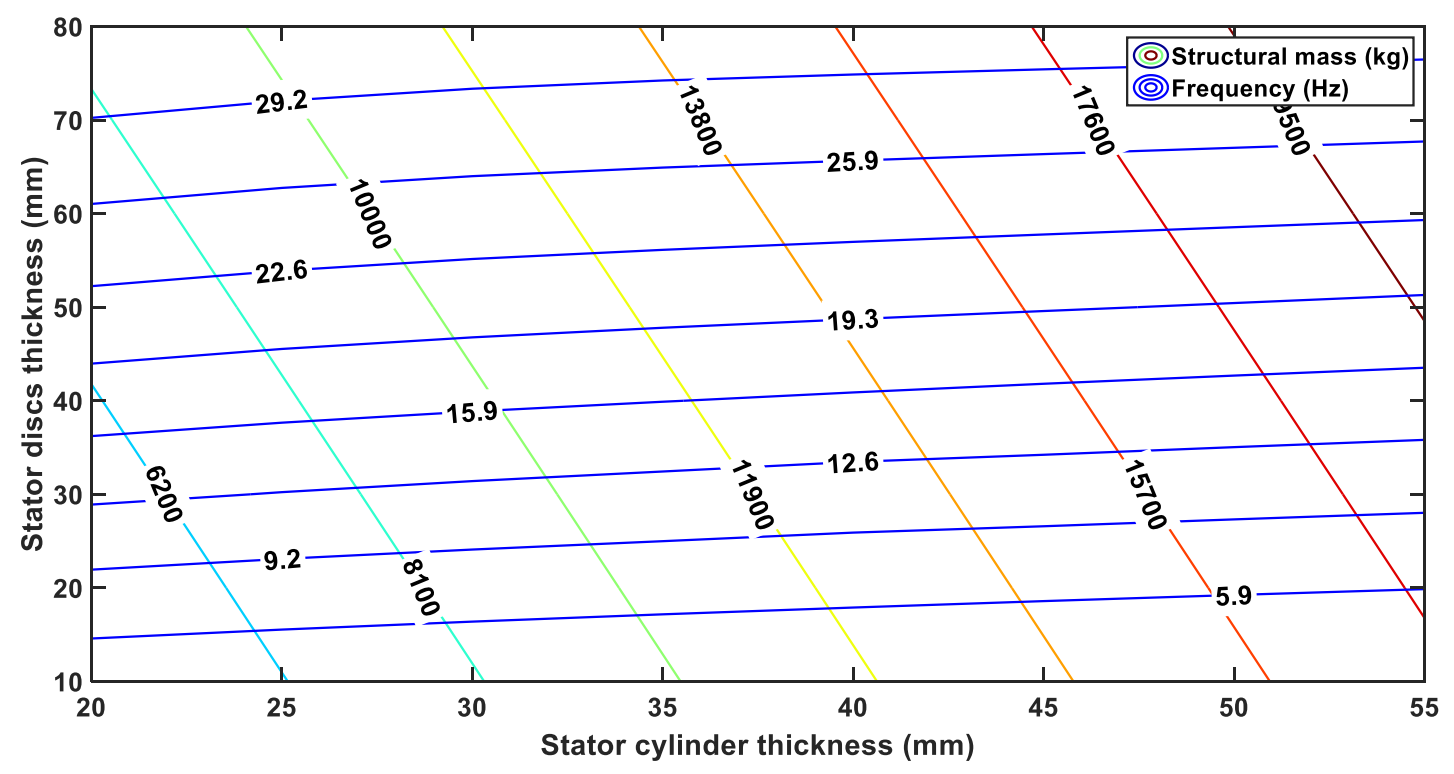

(b) 


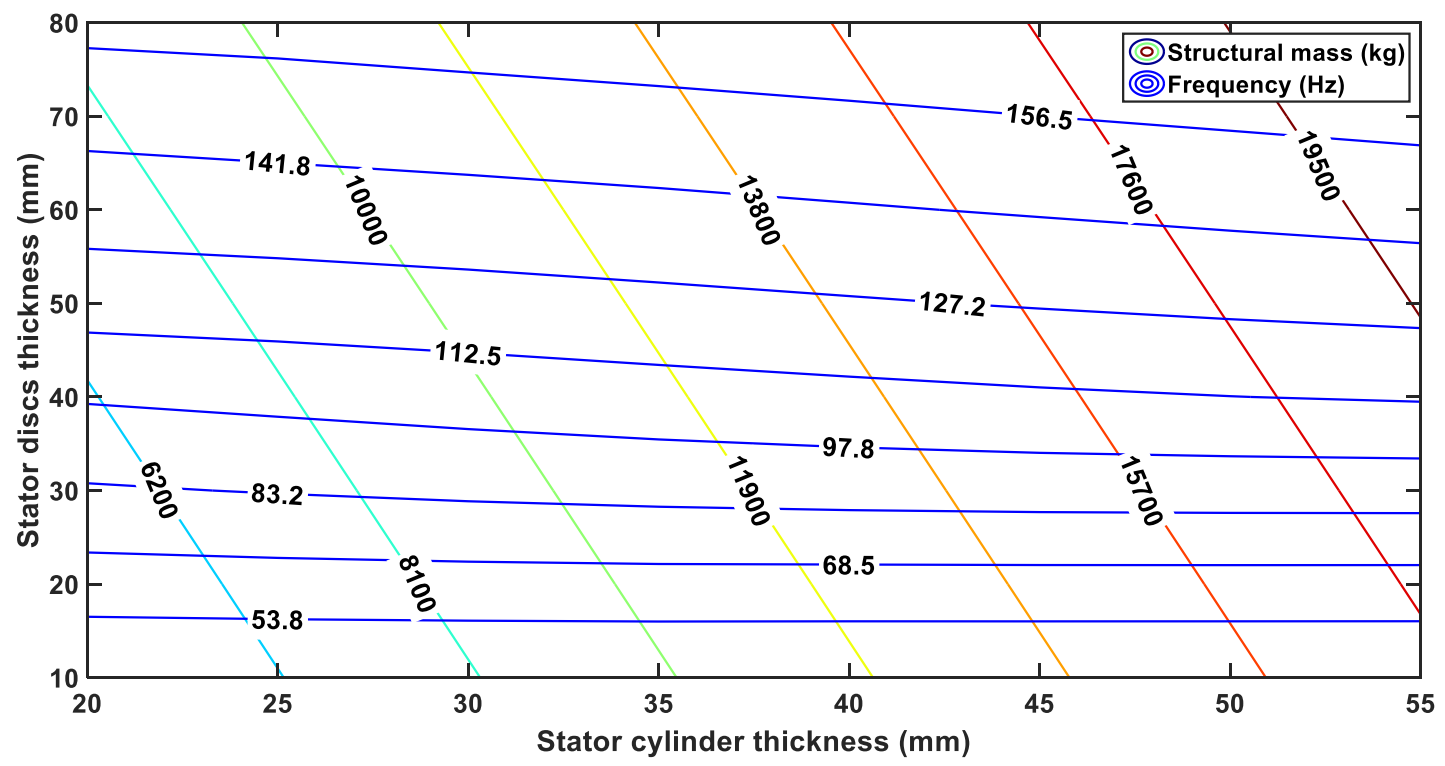

(c)

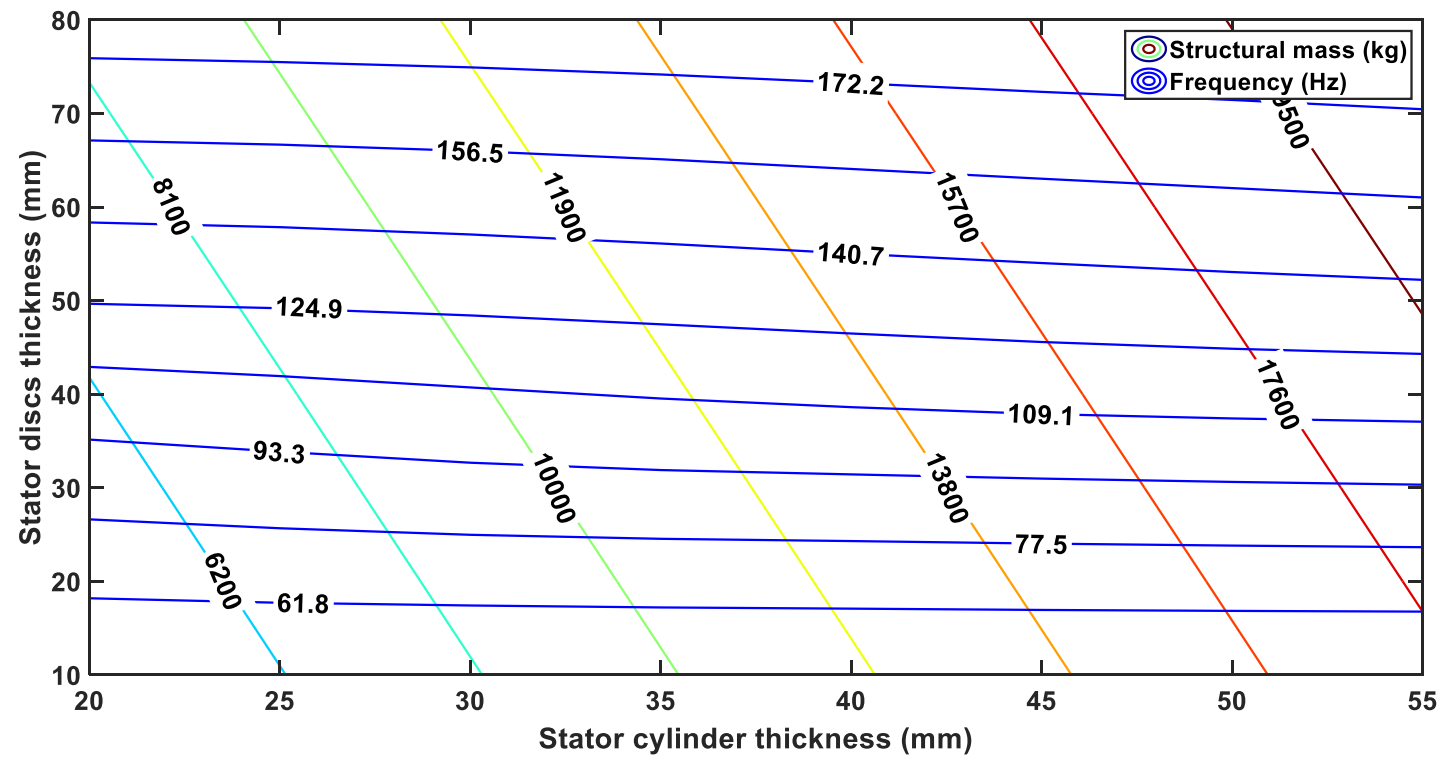

(d)

Figure 12 2D Optimisation for a $3 \mathrm{MW}$ electrical generator with natural frequencies criterion (a) $3^{\text {rd }}$ rotor mode; (b) $1^{\text {st }}$ stator mode; (c) $7^{\text {th }}$ stator mode; (d) $10^{\text {th }}$ stator mode

After optimising a disc generator structure by looking at it from a static viewpoint, one can assess its dynamic behaviour and estimate the structure natural frequencies by entering its dimensions in the contour plots given. With the information provided by this reliable and fast method and the resonant frequencies given, a Campbell diagram can be created and the best ways to maintain the machine's integrity can be identified. 


\subsection{Alteration of structure's natural frequencies by using stiffeners}

The evaluation of the structure's behaviour under resonance helps the design engineers to make decisions on how to alter the natural frequencies of the structures. For example, in the case where the disc deforms axially due to axial deflection of the cylinder sub-structure, the use of stiffeners providing some extra axial stiffness might help. A finite number of stiffeners of isosceles triangular shape with $60 \mathrm{~mm}$ of thickness, equally spaced, were implemented, in first instance, in the optimum rotor steel structure presented by Jaen-Sola in [18], which has a mass of $8,120 \mathrm{~kg}$ and the following dimensions: rotor disc thickness $t_{\mathrm{d}}=40 \mathrm{~mm}$, rotor cylinder thickness $t_{\mathrm{c}}=37.5 \mathrm{~mm}$; in order to quantify their effect on the structure natural frequencies. The total mass of the optimum generator (rotor and stator) was $17,571 \mathrm{~kg}$, with the stator having a thickness for the discs $t_{\mathrm{ds}}=20 \mathrm{~mm}$ and a cylinder thickness $t_{\mathrm{cs}}=33 \mathrm{~mm}$ [17].

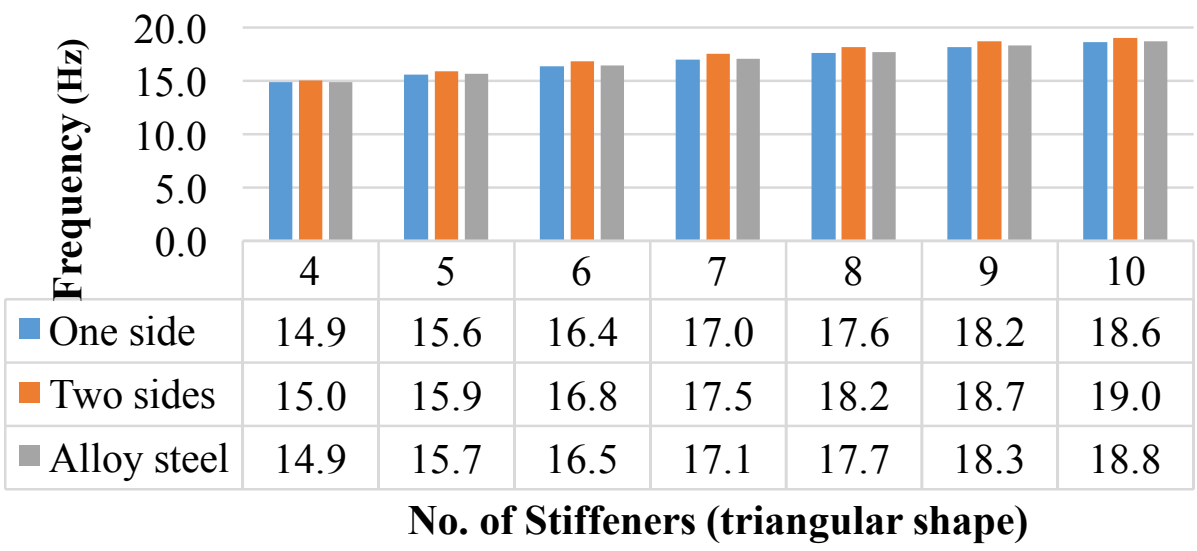

- One side $\quad$ Two sides $\quad$ Alloy steel

(a)

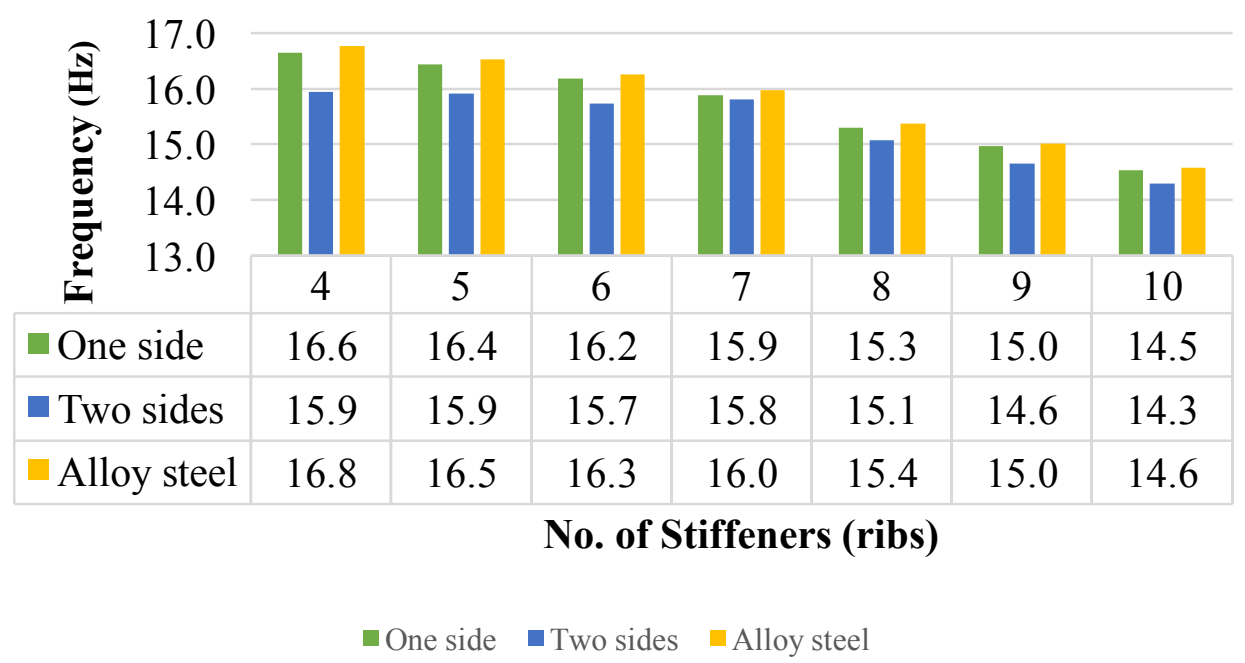

(b) 


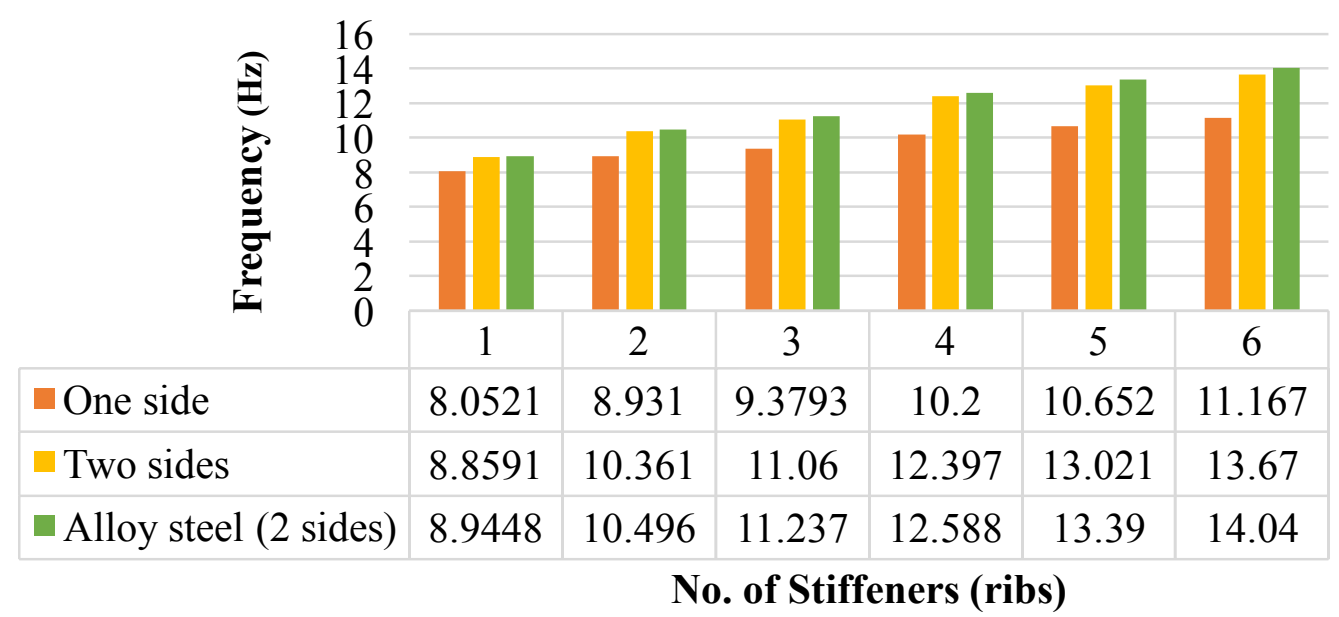

One side $\quad$ Two sides Alloy steel (2 sides)

(c)

Figure 13 (a) Quantification of the effect produced by the implementation of triangular stiffeners in the baseline rotor disc structure on the $3^{\text {rd }}$ mode shape; (b) Quantification of the effect produced by the implementation of rib stiffeners in the baseline rotor disc structure on the $3^{\text {rd }}$ mode shape; (c) Quantification of the effect produced by the implementation of rib stiffeners in the baseline stator disc structure on the $1^{\text {st }}$ mode shape

The investigation was started with a modal study of the structure without any stiffener. The result for the third mode shape was $13 \mathrm{~Hz}$. Then, 2 stiffeners were introduced on one side of the disc verifying that their effect on the structure natural frequencies was almost negligible although $164.6 \mathrm{~kg}$ were added to the overall mass. An unacceptable outcome was again obtained for 3 stiffeners; hence the data began to be taken into consideration after them. As shown in Figure 13(a), a different number of stiffeners located on one side first and on both sides second were considered. In addition, a less dense material such as alloy steel, with a density of $7,700 \mathrm{~kg} / \mathrm{m}^{3}$ was also assumed for the stiffeners with the main objective of reducing their mass.

It was understood that the structure's natural frequencies of any mode increase linearly with the number of stiffeners placed on one side of the disc. The same behaviour was observed when using stiffeners on both sides with slightly higher frequencies. The utilisation of stiffeners made of alloy steel on one side of the disc slightly enhanced the dynamic performance of the structure but the reduction in mass achieved was not very significant as each stiffener weighted $79.2 \mathrm{~kg}$ for $82.3 \mathrm{~kg}$ of a stiffener made of conventional structural steel. The data retrieved from the analyses showed that the introduction of stiffeners, either on one side or on both, pushed up the natural frequencies slightly more than $2 \mathrm{~Hz}$ (with 10 stiffeners). However, a mass of $823 \mathrm{~kg}$ had to be included for that. Similar results were achieved for the rest of the modes where small increases of the natural frequencies are obtained in exchange for large amounts of mass added. It was also observed that the implementation of this type of stiffener did not suppose the increment of the participation factor of any other mode in any direction. Similarly, the use of a different type of stiffener, in the form of a rib, was tested on the rotor structure giving the results 
shown in Figure 13(b). The dimensions of the ribs utilised in the study were: length =1,200mm; width $=150 \mathrm{~mm}$; thickness (in the axial direction) $=150 \mathrm{~mm}$. Two types of material were analysed again. Structural steel (AISI 304) with a density of $8,000 \mathrm{~kg} / \mathrm{m}^{3}$ and alloy steel with a density equal to $7,700 \mathrm{~kg} / \mathrm{m}^{3}$. The mass for a rib made of structural steel was $216 \mathrm{~kg}$, whereas the mass for a rib made of alloy steel was $208 \mathrm{~kg}$. By looking at the results presented in Figure 13(b) it is understood that the use of low number of ribs placed only one on side of the disc might help increasing the natural frequency of the structure if it is necessary. The use of alloy steel shows a slightly better performance in that sense if compared to its counterpart made of steel. On the other hand, the use of large number of ribs might help decreasing the natural frequencies although a large amount of mass would be introduced. It was understood that the introduction of triangular stiffeners did not alter the participation factor of any other resonant mode in any direction.

Last but not least, the use of ribs was tested on the stator structure retrieving the outcomes shown in Figure 13(c). This time, the ribs were located on the outer axial surface of one of discs first and second on the outer surfaces of both discs. Again, the use of ribs made of alloy steel was analysed. Figure 14 shows the result acquired from the study of the first stator mode shape. The deflection given is in $\mathrm{mm}$ and it is only for reference.
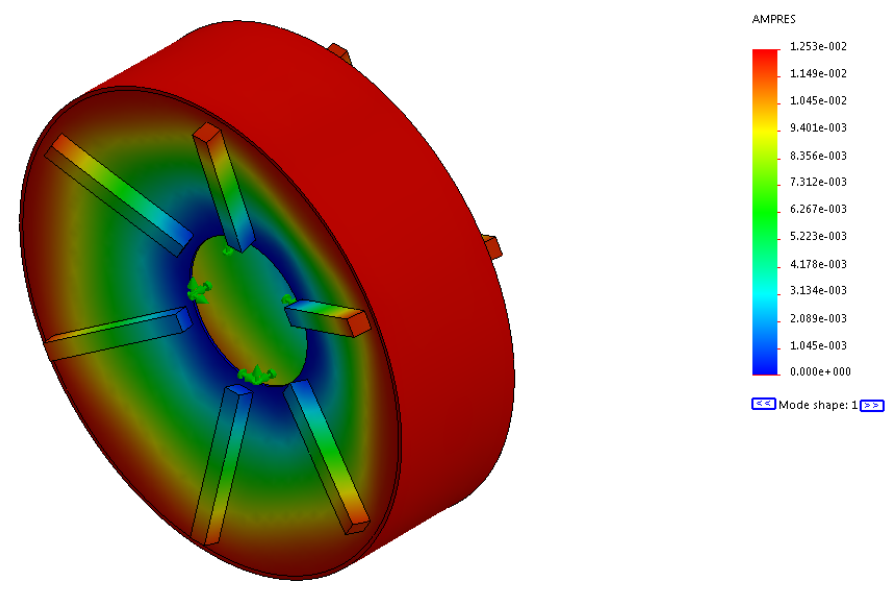

Figure $141^{\text {st }}$ stator mode shape with structural ribs on both discs

From the bar diagram, it can be seen how the use of ribs on both outer surfaces of the discs might help increase the natural frequencies of any mode considerably. Bearing in mind that the first mode shape without any stiffener corresponds to $7.13 \mathrm{~Hz}$, it is possible to double the frequency with the implementation of 6 ribs on both discs. That will suppose an augment in mass of 2,592kg. Moreover, it was observed that the participation factor could be modified. As well as mode 1, modes 7 and 10 were the most dangerous for the structure without any stiffener. However, with the implementation of 4 stiffeners made of structural steel on each disc, those modes lose their participation factor in detriment of mode 9. See Figure 15. The implementation of this particular number of stiffeners also changed the 
frequency range enough so that mode 9 , corresponding to $89.75 \mathrm{~Hz}$, is outside the influence zone of the electrical frequency in the Campbell diagram. This will allow us having a significant extension of the generator range of operation that will cover the highest wind speeds. With this improvement, the design becomes more suitable for offshore purposes. The total mass included is $1,728 \mathrm{~kg}$.

Frequency vs.Effective mass participation factor (EMPF)

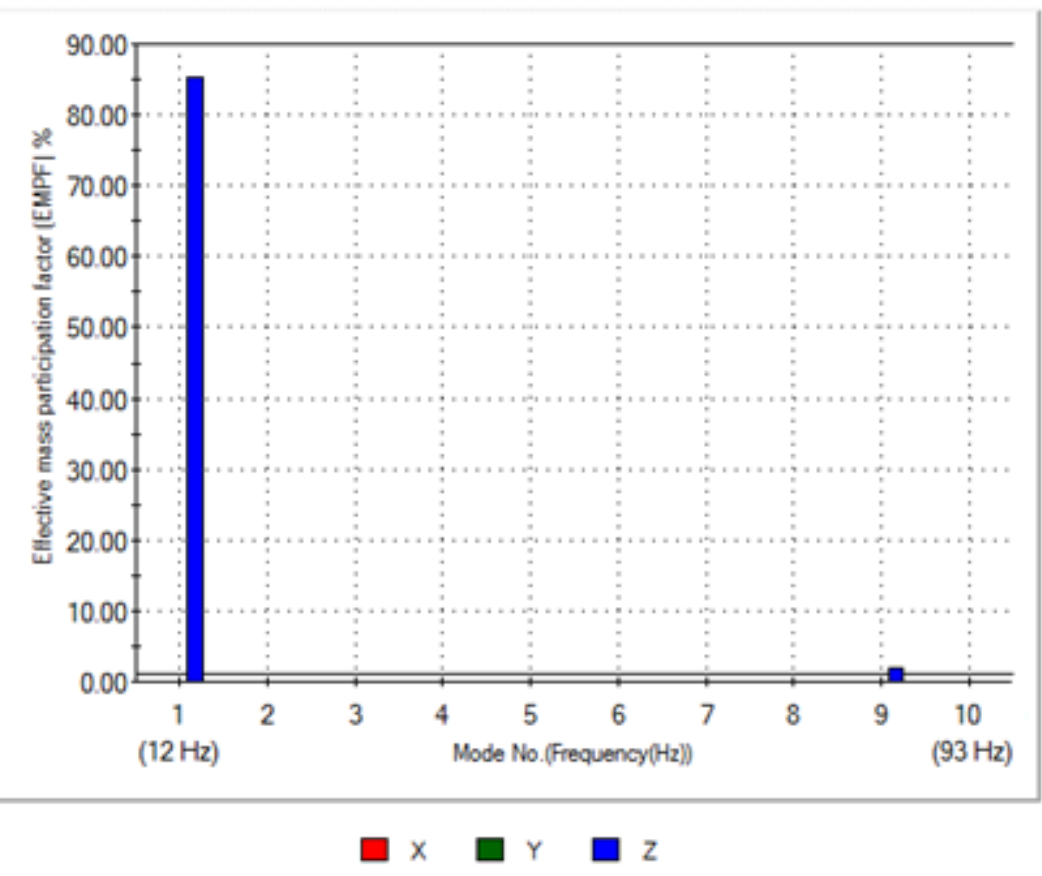

Figure 15 EMPF analysis of the stator structure with 4 structural steel stiffeners

\subsection{Conical structure optimisation using contour plots}

Having the disc structure deeply analysed, another type of structural configuration was looked at. As explained in Section 2, rotor conical structures include extra axial stiffness that disc structures miss, while keeping excellent radial stiffness characteristics. A rotor cone structure suitable for the proposed direct-drive machine has been optimised considering the deflection criterion described in [2], where deflection cannot be larger than $0.5 \mathrm{~mm}(10 \%$ of the airgap size $)$ in any direction, and also the structure natural frequencies that were found utilising finite element methods. The methodology can be summarised as:

First a cone structure was CAD modelled in SolidWorks. Random dimensions were picked for both the thicknesses of the cone and the cylinder sub-structures. Their values were 40 and $22 \mathrm{~mm}$, respectively. Figure 16(a) illustrates the rotor conical structure showing the dimensions as altered in the study. The angle ' $\psi$ ' is shown as well for clarification. After that, a set of static and dynamic analyses were run for each position and angle of the cone with the main aim of obtaining the mass, the maximum deflection and the first mode shape of the structures. 

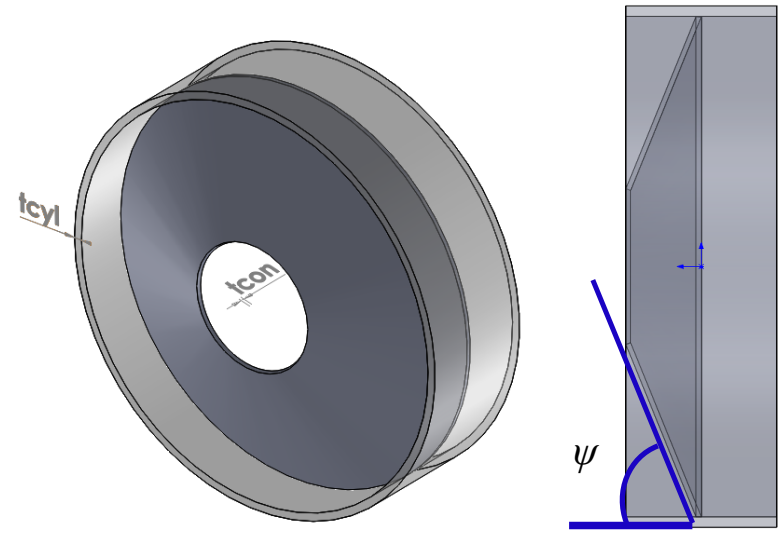

(a)

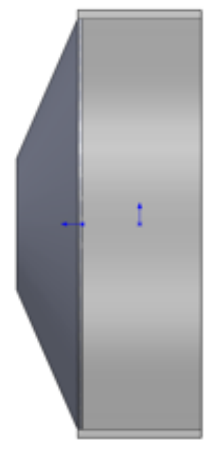

(1)

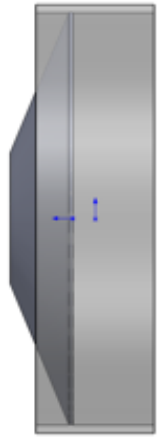

(2)

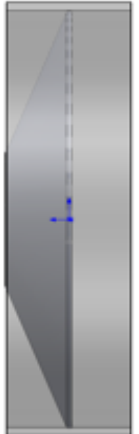

(3)

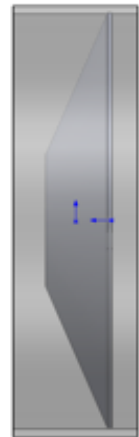

(4)

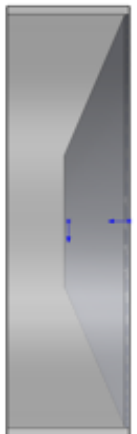

(5)

(b)

Figure 16 (a) Rotor conical structure as altered in the study; (b) View of the different positions for the cone substructure

Figure 17, illustrates how the structural mass of the conical rotor varies with angle. As seen, the larger the cone angle, the higher the mass. The quadratic curve fitted shows a parabola reaching its vertex at some point between 20 and 30 degrees before starting increasing again, bearing in mind that a disc is considered as a cone with 0 degrees angle. 


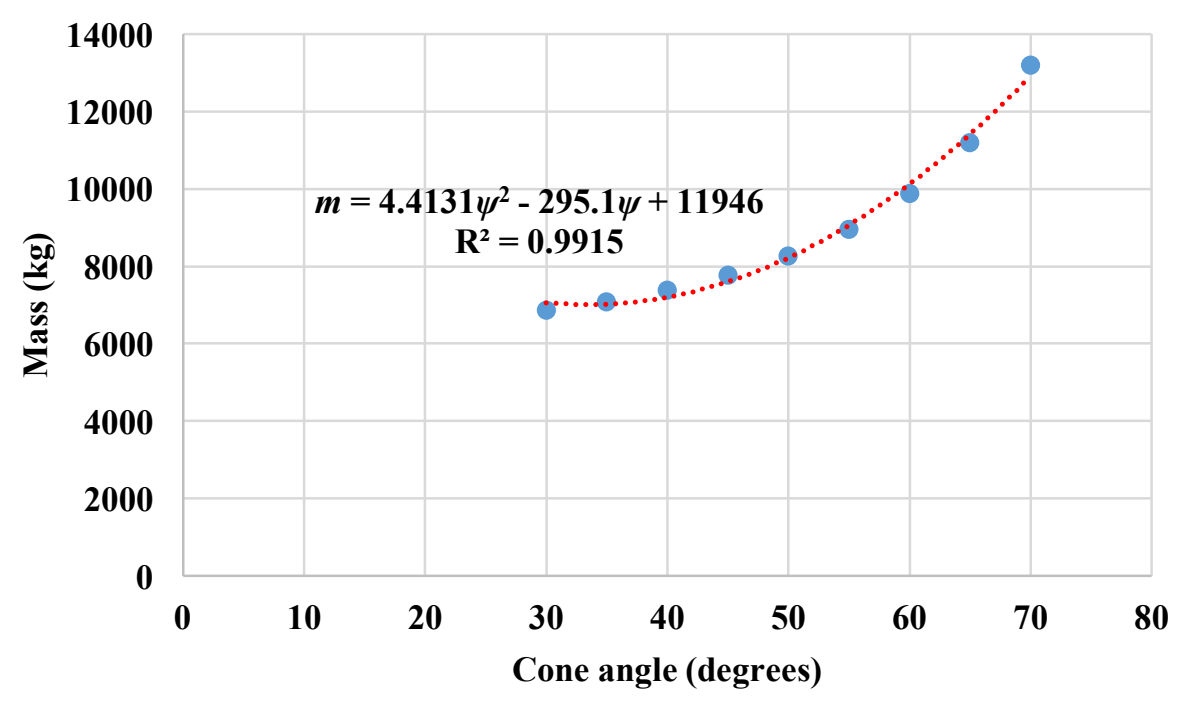

Figure 17 Rotor conical structural mass vs. cone angle

In Figure 19, a graphic 3D representation of the variation of the structural deflection ' $\delta$ ' and excitation frequencies ' $f$ ' with cone position and cone angle is given. Results obtained for cone angles between 0 and 20 degrees were not considered of interest as they were very similar to those achieved for the disc structure. As observed, the lowest structural deflections are acquired for cone positions 2 and 5 and between 30 and 50 degrees angles, whereas the highest natural frequencies for the first mode shape are obtained for positions 4 and 5 at around 50 degrees of angle. An effective mass participation factor study was made revealing that modes $7(41.18 \mathrm{~Hz})$ and $8(41.2 \mathrm{~Hz})$ were the most dangerous for a structure with the cone in the second position, whereas for a structure with the cone in the fifth position the most dangerous modes were $9(50.4 \mathrm{~Hz})$ and $10(50.42 \mathrm{~Hz})$. See Figure 18. It was not considered necessary to keep searching for modes with large EMPFs contributing to the dynamic response of the structure as the machine frequency spectrum has been already fully covered. Last mode studied here is mode 15 $(89 \mathrm{~Hz})$ and it is outside the influence zone of the electrical exciting frequency. 
Frequency vs.Effective mass participation factor (EMPF)

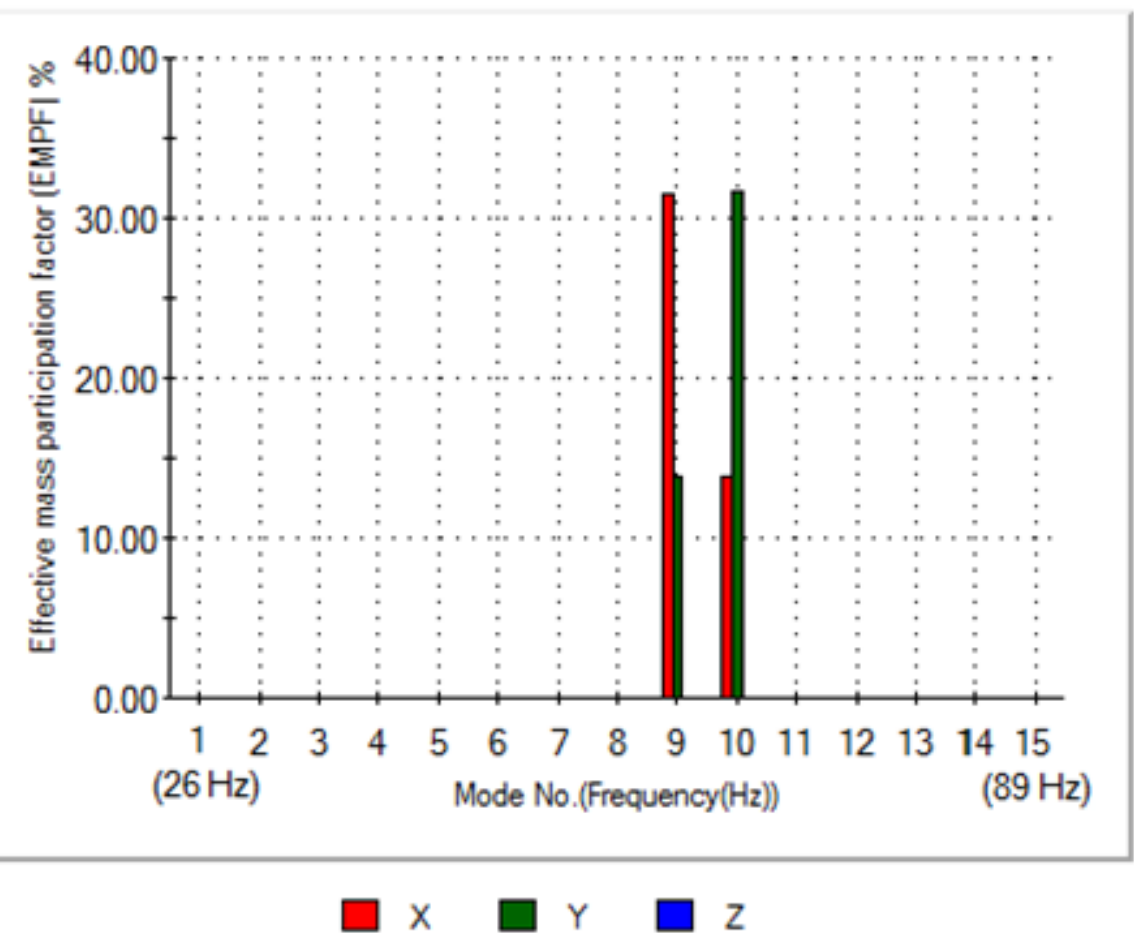

(a)
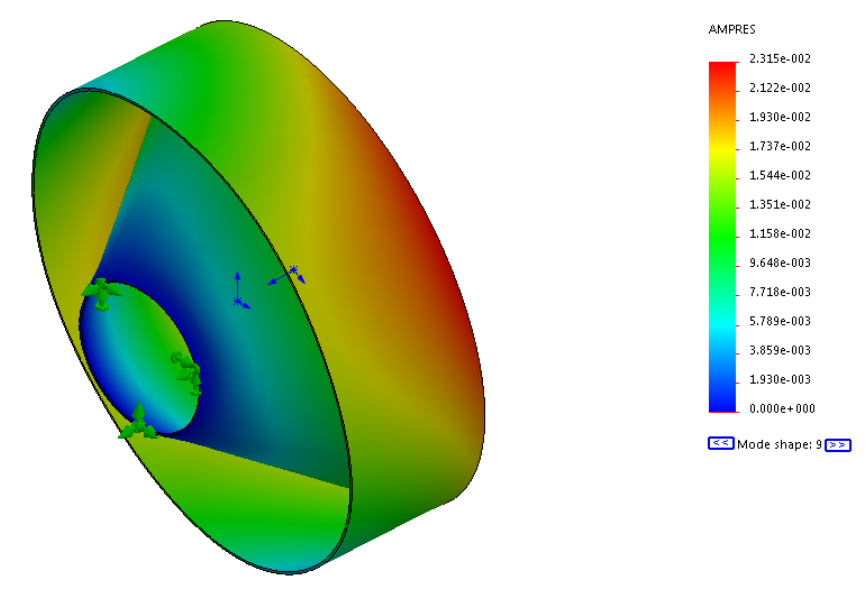

(b) 


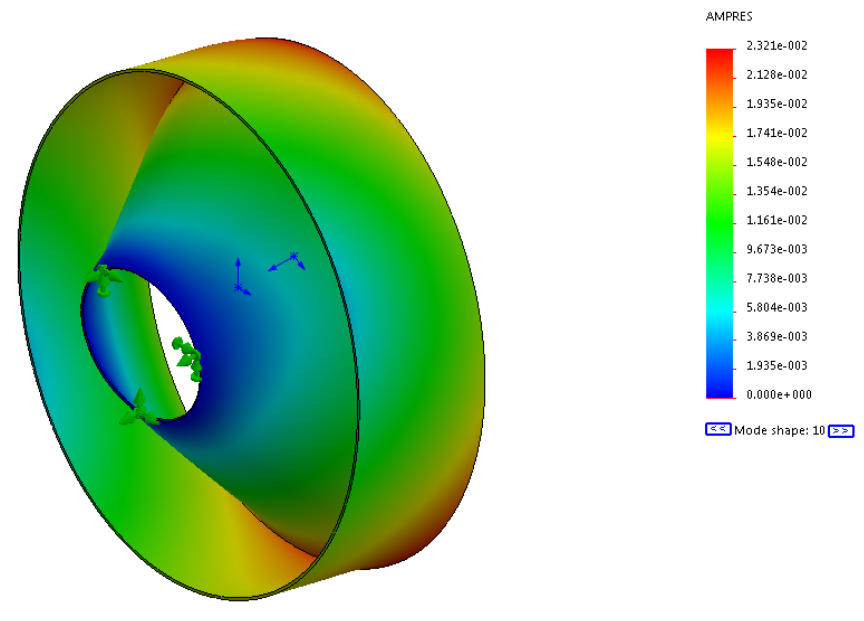

(c)

Figure 18 (a) Conical structure EMPF analysis; (b) Conical structure modal analysis: result for mode 9; (c) Conical structure modal analysis: result for mode 10

Figure 18(b) and (c) illustrate mode shapes 9 and 10 showing that the largest deflections take place on the rim. Considering that the structure is weaker in directions $\mathrm{X}$ and $\mathrm{Y}$ and by looking at the results for the mode shapes, it is possible to find the different options that can be adopted in order to change the rotor natural frequencies. Based on these observations and bearing in mind that the best layout needs to have the largest range of operation and lowest mass; it was considered that position 5 with a cone angle of 50 degrees was the most suitable configuration for an optimum structure.

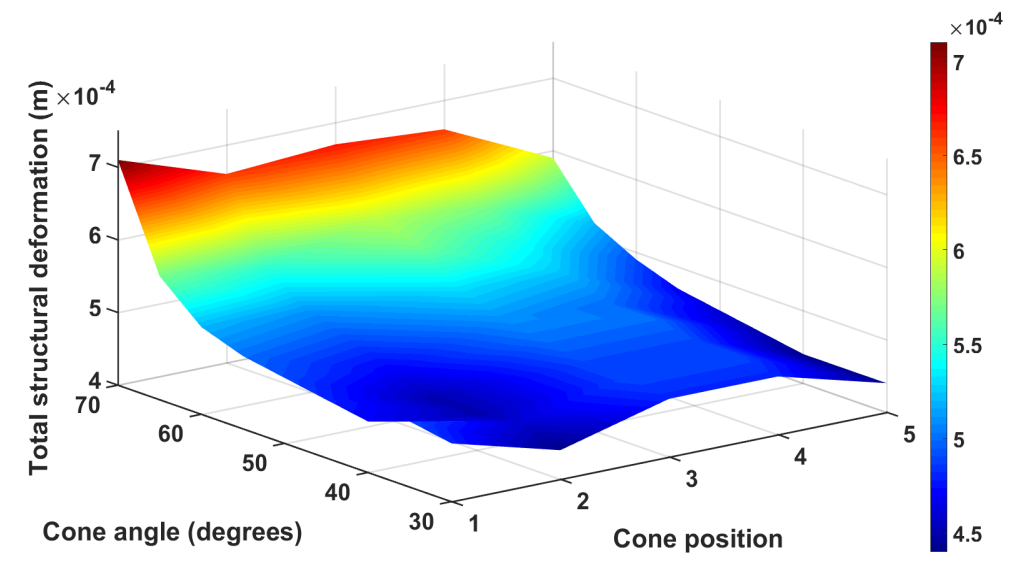

(a) 


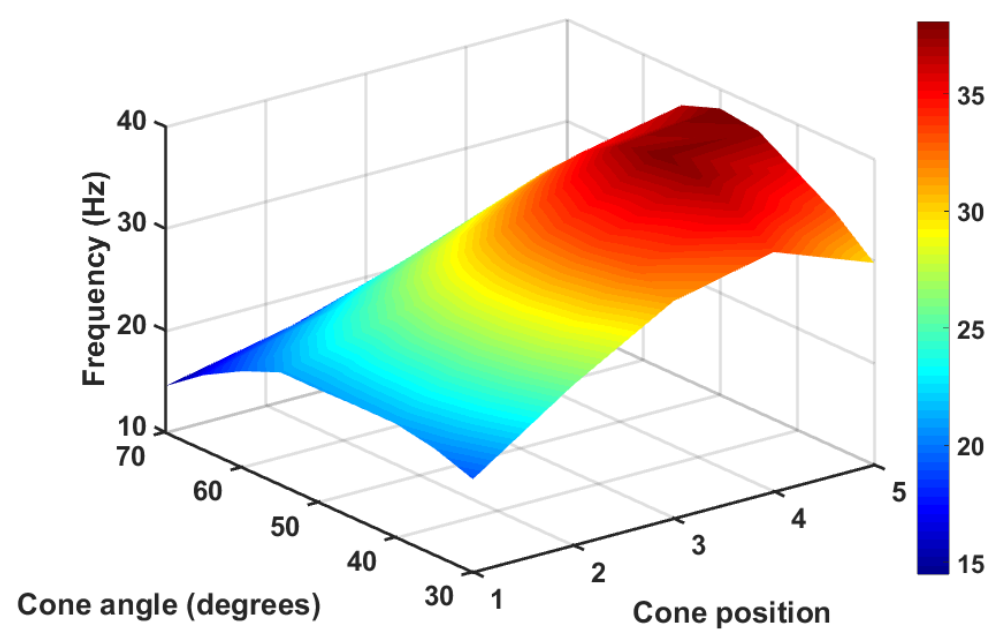

(b)

Figure 19 3D representation of the structural deflection (a) and frequency (b) variation with cone position and angle

Then, it was checked which sub-structure put more weight into the overall structure when adding the same thickness $(5 \mathrm{~mm})$. It could be understood that the cone sub-structure included $87 \mathrm{~kg}$ more than the cylinder. With the targeted sub-structure found, its thickness was diminished while the thickness of the cylinder was kept constant achieving a final result of $17 \mathrm{~mm}$ thickness for the cone and $22 \mathrm{~mm}$ for the cylinder. That yielded a total mass of $5,062 \mathrm{~kg}$ and a radial deflection of $4.827 \times 10^{-4} \mathrm{~m}$. Tangential and axial deflections were also checked observing that they were still within the limits (deflection cannot exceed $10 \%$ of the airgap size, which means $0.5 \mathrm{~mm}$, in any direction).

Once the optimum thicknesses were found, a sanity check was carried out obtaining that $50^{\circ}$ cone angle at position 5 was still the best layout.

With the finest configuration encountered, the dimensions of the rotor, ' $t_{\text {con }}$ ' and ' $t_{\text {cyl }}$, were altered and modal analyses were run in order to quantify the effect of increasing the thicknesses of the sub-structures on the overall frequency. The evaluated results are displayed in the contour plots given in Figure 20 for mode shapes 9 and 10. Through the analysis of the EMPF it could be seen that the alteration of the substructures thicknesses also changed the participation factor of the mode shapes, thus it is crucial to double-check the structure after finishing its design using the contour plot provided. The contour plots for the first 8 natural frequencies of the structure are given in the Appendix. 


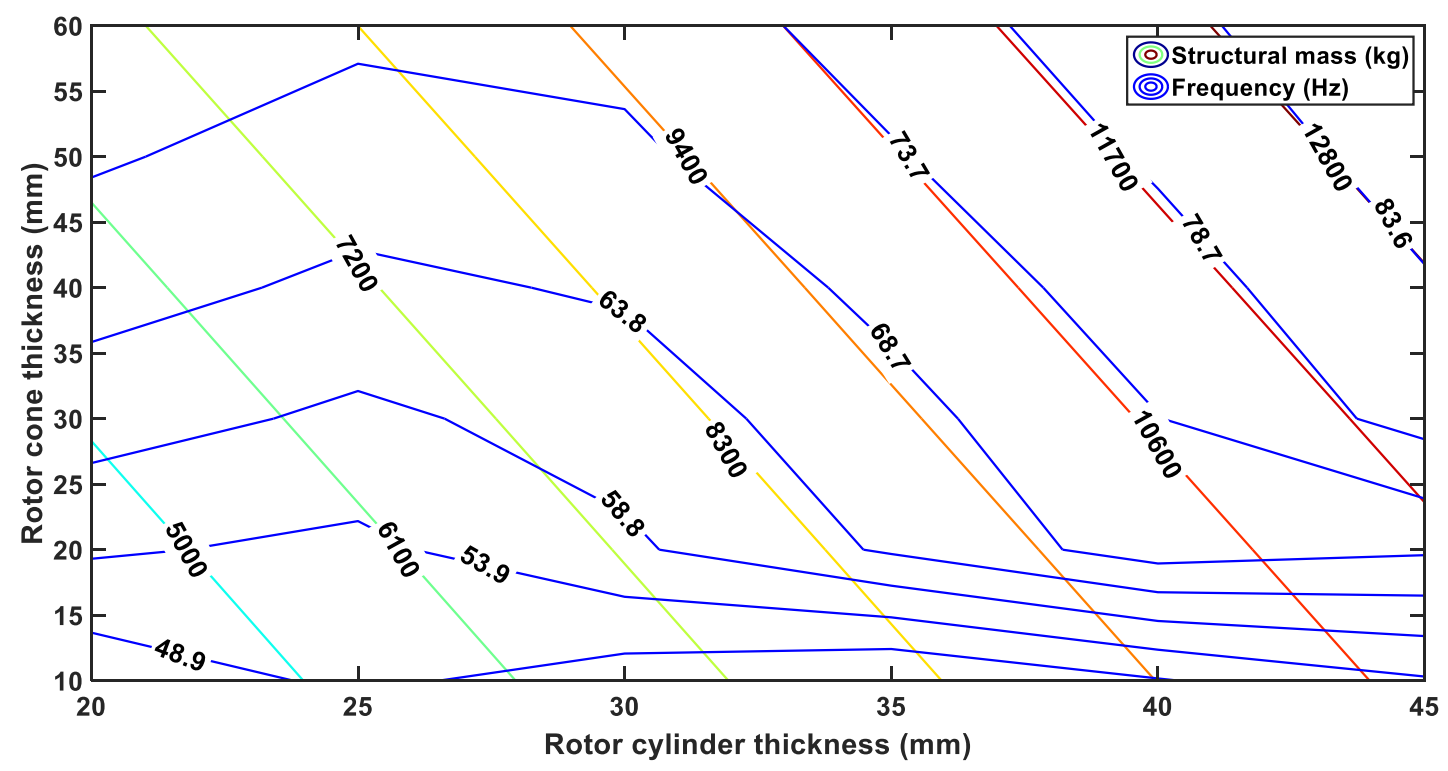

(a)

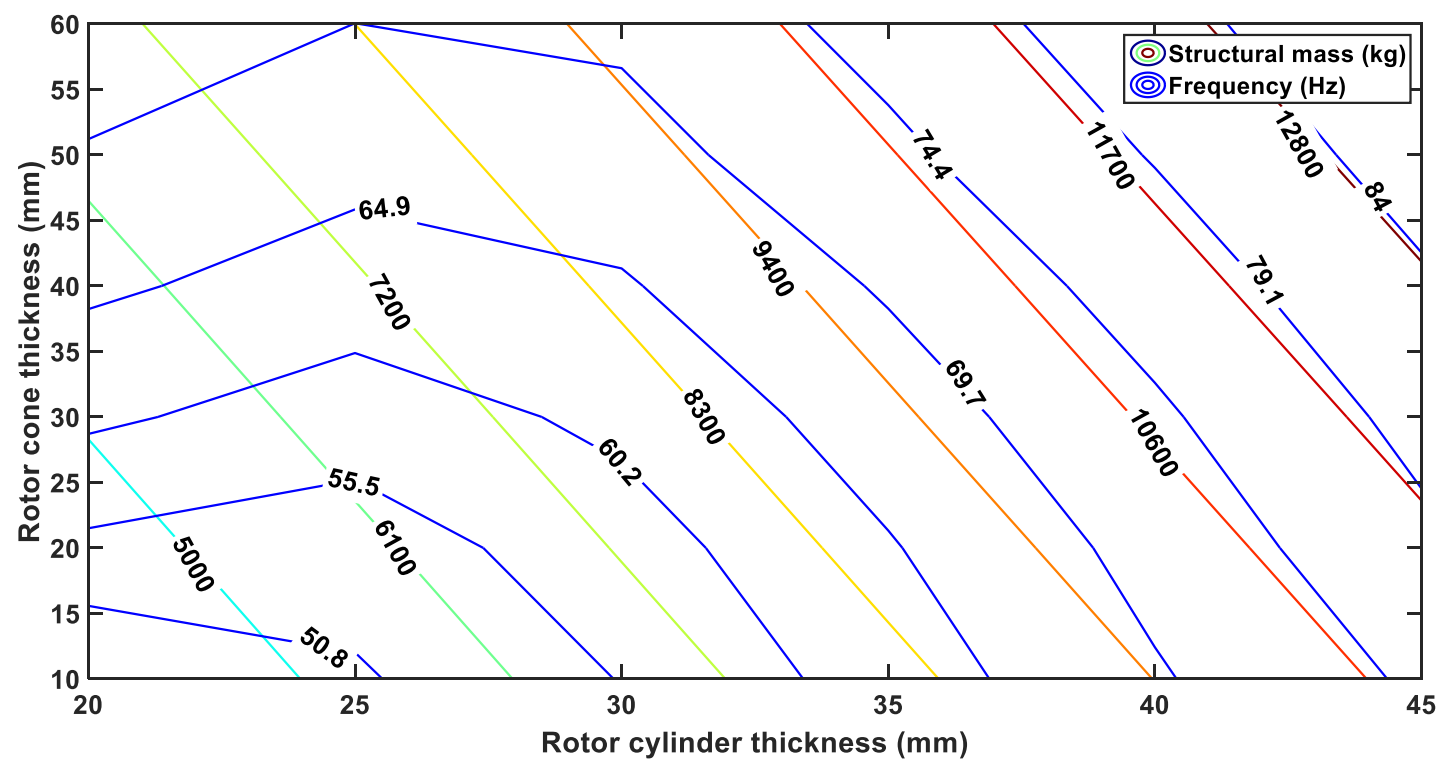

(b)

Figure 20 2D optimization of the rotor conical structure with natural frequencies criterion (a) Mode 9; (b) Mode 10

\section{Discussion}

Three distinct types of structural arrangements have been analysed in this paper. With the main aim of maximizing the electrical generator operation range while keeping its structural mass to a minimum a useful tool developed for disc structures to help the engineers during the early phase of their dynamic design has been presented. It consists of contour plots where the natural frequencies and the structural mass vary with thicknesses of the sub-structures forming the generator components. As seen in Figure 21 , with the utilisation of these contour plots the frequency of the third mode of the rotor structure can 
be decreased (blue arrow) by augmenting the cylinder thickness. This will help us gather more energy at low wind speeds. In the stator case, mode 1, 7 and 10, which are the most dangerous according to the effective mass participation factor study, can be adjusted by increasing the discs thicknesses. As the third rotor mode shape is at $13 \mathrm{~Hz}$, there is still room for the first stator mode $(7.13 \mathrm{~Hz})$ to be pushed up and with it modes 7 and 10 (black arrows). An EMPF analysis showed that the variation of substructures thickness did not suppose any change in the effective mass participation factor of any other mode, rather than the dominant ones where small variations happen, in any direction.

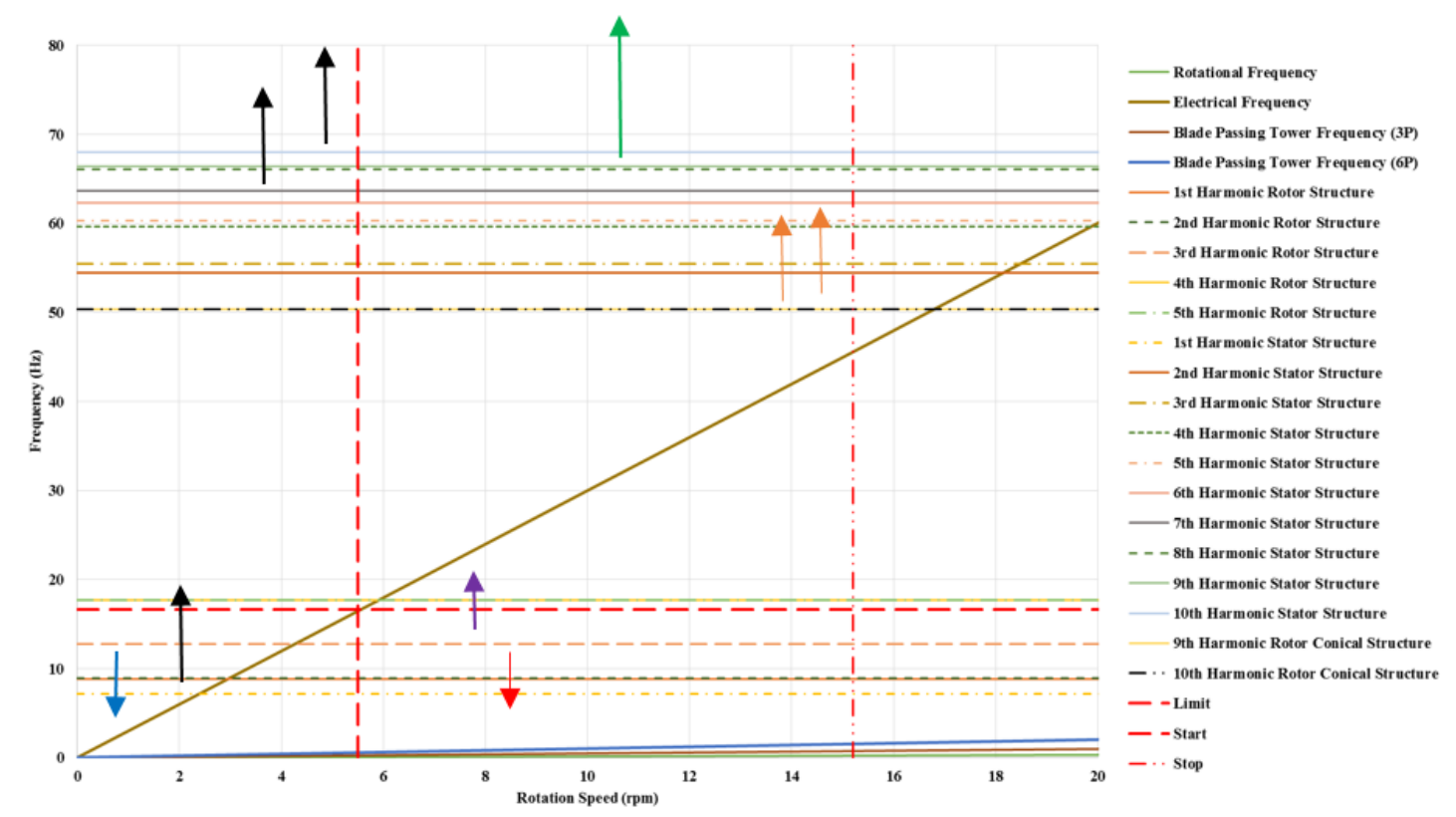

Figure 21 Campbell diagram showing the effect caused by the utilisation of the different proposed techniques

Alternatively, the use of stiffeners has been introduced as a potential solution to change the natural frequencies of the structures without sensibly augmenting their mass. A series of modal analyses were carried out over the baseline structure model having two different types of stiffeners attached and equally spaced. Firstly, triangular stiffeners were placed on one side of the rotor disc sub-structure and then on both sides. It was understood that the utilisation of this sort of stiffeners puts too much weight $(82.3 \mathrm{~kg}$ per stiffener) on the structure while the third natural frequency only increases by around $0.6 \mathrm{~Hz}$ per stiffener (purple arrow). However, it was observed that the use of stiffeners on only one side was much more efficient than on both sides in terms of frequency and mass. For this reason, it was thought that a less dense material, such as alloy steel, could be employed to further reduce the mass of such layout. The results obtained from the modal studies revealed that the addition of structural mass was still high. After an EMPF study it could be seen that the introduction of this type of stiffeners did not change the participating mass of any other mode shape, apart from the dominant where small deviations take place, in any direction. 
A rib type of stiffener was the second type of stiffener tested. First the rotor structure was analysed having ribs on only one side of the disc sub-structure and second on both sides. Alloy steel was again tried as an approach to further reduce the system's mass. It was understood that the use of rib stiffeners on one side gave better frequency results than placing them on both sides. Stiffeners made of alloy steel showed slightly better performance in this sense. It was also seen that the use of a high number of ribs could be employed to decrease the frequency of the third mode of the rotor (red arrow). Nonetheless, that would imply the introduction of a large amount of mass $(2,160 \mathrm{~kg}$ per 10 stiffeners $)$. Once again, the effective mass participation factor analysis revealed that no change takes place in the mass participating in other modes, except for the dominant where small variations happen, and in any direction.

The use of ribs was finally tested on the stator structure. First on the outer axial surface of one of the discs and second on the outer surfaces of both discs. Alloy steel was tried for the ribs too in order to minimize their mass. The modal studies showed that the use of this type of stiffeners is effective when altering the natural frequencies of the stator that nearly goes up by $1 \mathrm{~Hz}$ per stiffener. In this case, the most important fact to be considered was not the variation in frequency but the change in the EMPF of the dominant modes. Mode 1 was still the dominant mode. However, by implementing the 4 ribs on each disc, modes 7 and 10 lost influence in detriment of mode 9. Moreover, the introduction of the stiffeners modified the frequency spectrum so that mode 9 goes from $66.41 \mathrm{~Hz}$ without stiffeners to $89.75 \mathrm{~Hz}$ with 4 stiffeners on each side (green arrow). This means, that mode 9 would out of the influence zone of the electrical exciting frequency what allows us to gather energy at the highest wind speeds without taking any risk.

On the other hand, rotor conical structures were assumed. Attracted by their good axial stiffness features, a conical structure was optimised with a view of minimising its mass considering deflection limitations and the frequencies of the lowest mode shapes. It was found that a rotor structure with a cone angle of $50^{\circ}$ placed at the 5 th position with $17 \mathrm{~mm}$ thickness for the cone and $22 \mathrm{~mm}$ for the cylinder presented the best outcomes. This yielded a total rotor mass of 5,062kg. Contour plots for the first 10 natural frequencies of the rotor conical structure were obtained and EMPF study carried out, which revealed that modes 9 and 10 are the most dangerous. In order to avoid the mentioned modes the cone thickness can be increased. This will put up the frequencies of the dangerous modes as the orange arrows state in the Campbell diagram. During the structure analysis it was observed that effective mass participation factors vary and change from mode to mode and from direction to direction. Because of that it is recommended that the optimisation of such structure needs to be done paying special attention to the changes taking place in the EMPF analysis as the structure behaviour is much more variable than that of the disc structure where the dominant modes are by far mode 3 for the rotor and mode 1 for the stator. By checking the Campbell diagram presented in this Section, it can be seen that the most dangerous rotor mode is the third, whereas for the stator these are mode 1, 7 and 10. In order to avoid them, it would be necessary to have the electrical machine working in a range between 5.5 and $15.2 \mathrm{rpm}$. This 
means that the wind energy that could be harvested below 5.5rpm and over $15.2 \mathrm{rpm}$ is lost. Potential solutions have been given here to approach the problem. For instance, with the variation of the rotor cylinder thickness, it would be possible to decrease the frequency of mode 3 allowing more energy gathering at low wind speeds although this would add some extra mass to the system that would depend on the amount of energy that wants to be harvested at low wind speeds. Nonetheless, as wind speeds are higher and the conditions are steadier offshore it is vital to gather as much as energy as possible at high wind speeds. For that, the use of a stator with 4 rib stiffeners attached to the outer faces of each disc is proposed as it would give us the possibility to push the stop line presented in the diagram from 15.2 to $20 \mathrm{rpm}$, which corresponds to the cut off wind speed $(24 \mathrm{~m} / \mathrm{s})$. That would represent the addition of $1,728 \mathrm{~kg}$ to the system. The use of conical structures with larger cone thicknesses would be another possibility as they are significantly lighter compared to disc structures working under the same static conditions $(5,062 \mathrm{~kg}$ vs. $8,120 \mathrm{~kg})$ although its design must be more concise as the variations in the EMPFs is much more dynamic than those of disc structures.

The last solution that can be noticed by having a look at the said diagram goes through altering the number of pole pairs so that the slope of the electrical frequency line can be changed. With a higher number of poles, the inclination of the line would go down interfering with the lowest rotor and stator modes at higher rotational speeds which would force us to narrow down the machine operating range. In addition, saturation problems could arise. But, if the number of poles is decreased, the slope of the line would go up giving us more room for machine operation. Nonetheless, it is important to notice that by doing so the highest stator modes could get excited at lower rotational speeds. Also, with lower number of poles thicker rotor and stator yokes would be necessary which would make the machine heavier. Exploration of the rotor and stator interference and bearing features and location have not been considered in this analysis.

Another feature to bear in mind are short circuits. During a short circuit event (they can create 6-7 times of the rated torque), the magnetic field could react causing rotor eccentricity, transferring the entire load from the generator structure to the bearing. Moreover, the excitation of the structural modes can generate changes in the inductance of the coils introducing higher harmonics in the currents. Therefore, it would be necessary to introduce a model of the bearing able to replicate their characteristic non-linear behaviour so as to further increase the accuracy of the study.

The dynamic behaviour of the structure has been studied and tried to improve according to the requirements demanded by an offshore wind turbine. Several techniques, which are considered valid for disc and conical structures, have been described and verified here. A trade-off process between frequencies, EMPF and structural mass is necessary to complete the generator structural optimisation. The results achieved were filtered until finding the appropriate data that could be useful for a design engineer looking at this type of devices. Note that no external influence was considered in these studies. 


\section{Conclusions}

A useful instrument for the dynamic design of disc structures has been presented. The use of the provided contour plots allows the engineer to make accurate estimations of the weight and the natural frequencies of the structure to be produced. Later on, the introduction of stiffeners was proposed with the main aim of altering the structure natural frequencies without adding too much weight. The best results were obtained by placing the stiffeners on both sides of the stator discs sub-structure which allow us to avoid the highest stator mode shapes. This makes the gathering of energy at high wind speeds.

Due to its potentially good radial and axial stiffness characteristics, a rotor conical structure was proposed. The authors believe that conical structures should be taken into consideration when approaching the design of a generator structure due to their outstanding static and dynamic characteristics. Nevertheless more research would be necessary as its design presents more challenges than disc structures with more variables influencing the optimisation, such as the cone position and angle, and big variations in the effective mass participating factors. The introduction of stiffeners should also be tested on conical structures. It is believe that with the use of the already described methods and proposed materials, with lower density, the mass of these structures can be further optimised. The use topology optimization tools could be of interest as well. The removal of certain zones carrying no loads can help decrease the structure's mass and in some cases increase its stiffness at the same time. Commercial pieces of software, such as SolidWorks, do not allow the user to consider the natural frequencies and the EMPFs simultaneously in the optimisation study. Therefore, the final stage of the process has to be carried out by hand. Finally, making use of composite materials should also be considered as the stiffness of the structure in any direction can be tailored and the density of the material is much lower than that of steel. In this case the higher price of manufacturing and material cost would become variables that would acquire much more weight in the design study. A trade-off process between frequencies, EMPF and structural mass is necessary to complete the generator structural optimisation.

\section{Acknowledgements}

We would like to thank Professor David Infield and Dr Ozan Keysan from the Electronic and Electrical Engineering departments of the University of Strathclyde, in Glasgow (UK), and the Middle East Technical University, in Ankara (Turkey), respectively, for providing valuable feedback on this article. Funding: This work was supported by the EPSRC Wind and Marine Energy Systems Centre for Doctoral Training in the University of Strathclyde.

\section{References}

[1] H. Polinder, SWH De Haan, M. R. Dubois, et al., "Basic operation principles and electrical conversion systems of wind turbines", EPE J., 2005, 15, (4), pp. 43-50. 
[2] P. Jaen-Sola and A. S. McDonald, "Structural Analysis and Characterization of Radial Flux PM Generators for Direct-Drive Wind Turbines," 3rd Renew. Power Gener. Conf. (RPG 2014), Naples (Italy) Sept. 2014.

[3] T. Hartkopf, M. Hoffmann, S. Jockel, "Direct-drive generators for megawatt wind turbines", Proc. European Wind Energy Conf., Dublin, Ireland, October 1997, pp. 668-671.

[4] M. A. Mueller, A. S. McDonald, D. E. MacPherson, "Structural analysis of low-speed axialflux permanent-magnet machines", IEE Proc. Electrc. Power Appl., 2005, 152, (6), pp. 1417-1426.

[5] A. S. McDonald, "Structural analysis of low-speed, high torque electrical generators for direct drive renewable energy converters", Ph.D. thesis, School of Engineering \& Electronics, University of Edinburgh, Edinburgh (UK), 2008.

[6] P. Jaen-Sola and A. S. McDonald, "A Comparative Study of Methods for Modelling the Structural Stiffness of Generator Components", IET Power Electronics, Machines \& Drives (PEMD), Glasgow (UK), Apr. 2016.

[7] L. Sethuraman, "Hydrodynamics and drive-train dynamics of a direct-drive floating wind turbine", Ph.D. thesis, University of Edinburgh, Edinburgh (UK), 2014.

[8] Y. Xing, M. Karimirad, T. Moan, "Effect of Spar-Type Floating Wind Turbine Nacelle Motion on Drivetrain Dynamics", In Proceedings of EWEA 2012 Annual Event, Copenhagen, Denmark, 16-19 April 2012.

[9] Y. Xing, M. Karimirad, T. Moan, "Modelling and analysis of floating spar-type wind turbine drivetrain", Wind Energy, (2012).(DOI: 10.1002/we.1590), pp.1-23.

[10] M. Kirschneck, "Mastering Electro-Mechanical Dynamics of Large Off-Shore Direct-Drive Wind Turbine Generators”, Ph.D. thesis, TU Delft, Delft (The Netherlands), 2016.

[11] ISO 10816-21:2015(en) Mechanical vibration — Evaluation of machine vibration by measurements on non-rotating parts — Part 21: Horizontal axis wind turbines with gearbox

[12] https://www.gerenewableenergy.com/wind-energy/technology/pure-torque, [Online] Last Accessed 22/02/2018.

[13] A. Zavvos, "Structural Optimisation of Permanent Magnet Direct Drive Generators for 5MW Wind Turbines”, Ph.D. thesis, University of Edinburgh, Edinburgh (UK), 2013.

[14] M.J.N. Priestley, F. Seible, and G.M. Calvi., Seismic Design and Retrofit of Bridges, John Wiley \& Sons (1996).

[15] SolidWorks help file, Frequency Analysis Graph Property Manager, http://help.solidworks.com/2015/english/solidworks/cworks/hidd frequency analysis graph.htm,

[Online] Last Accessed 17/04/2018.

[16] GH James, TG Carne, JP Lauffer, AR Nord., "Modal Testing Using Natural Excitation”. Proceedings of the 10th International Modal Analysis Conference (IMAC), San Diego, California.1992. p. 1209-16.

[17] J. N. Stander, G. Venter, and M. J. Kamper, "Review of direct-drive radial flux wind turbine generator mechanical design", Wind Energy, Wiley, vol. 15, (3), pp. 459-472, Apr. 2012.

[18] P. Jaen-Sola, "Advanced Structural Modelling and Design of Wind Turbine Electrical Generators”, Ph.D. thesis, Wind Energy Systems CDT, University of Strathclyde, Glasgow (UK), 2017.

[19] SolidWorks, http://www.solidworks.com/, [Online] Last Accessed 22/02/2018. 
[20] Alstom/GE renewable energy, https://www.gerenewableenergy.com/windenergy/turbines/offshore-turbine-haliade, [Online] Last Accessed 22/02/2018.

[21] ESM Gmbh, https://www.esm-gmbh.de/EN/Products/Couplings, [Online] Last Accessed 22/02/2018.

[22] University of Alberta, https://sites.ualberta.ca/ yousefim/bfiles/Ebooks/Vinogradov\%20$\%$ 20Fundamentals $\% 20$ of $\% 20$ kinematics $\% 20$ and $\% 20$ dynamic $\% 20$ of $\% 20$ machines $\% 20$ and $\% 20$ mecha nisms.pdf, [Online] Last Accessed 22/02/2018.

[23] Hawkridge systems, https://www.hawkridgesys.com/blog/simulation-adaptive-meshing/ [Online] Last Accessed 22/02/2018.

[24] Shrestha et al., "Structural Flexibility: A Solution for Weight Reduction of Large Direct-Drive Wind-Turbine Generators", IEEE Transactions on Energy Conversion, Vol. 25, No. 3, Sept. 2010.

[25] SolidWorks, http://www.solidworks.com/sw/products/simulation/frequency-analysis.htm, [Online] Last Accessed 22/02/2018.

[26] F. De'nan, C. K. Keong, N. S. Hashim, and K. J. Yan, "Parametric study on shear behaviour for triangular web profile steel section with openings", AIP Conference Proceedings, Vol. 1892, Issue 1., October 2017. 


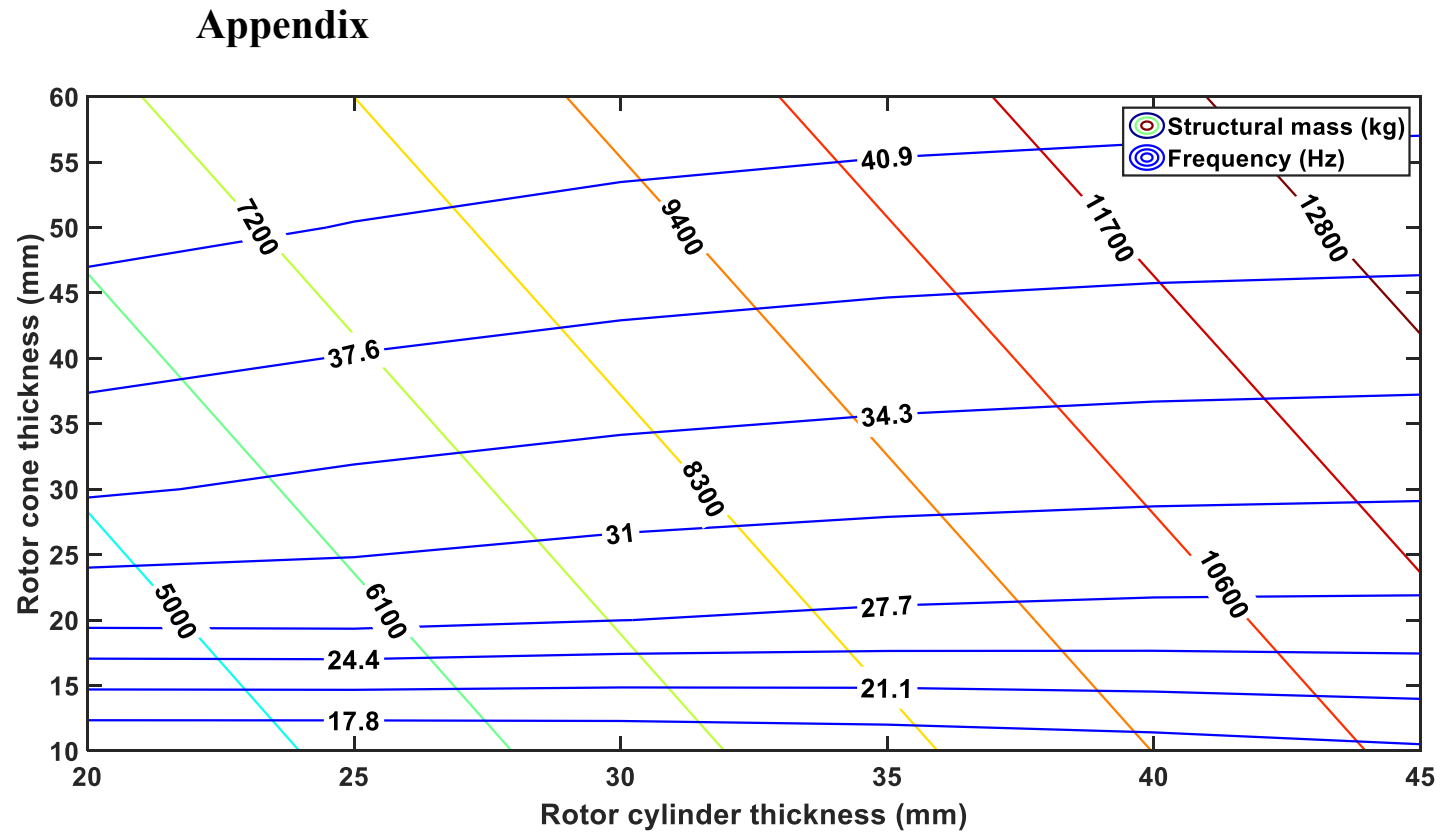

(a)

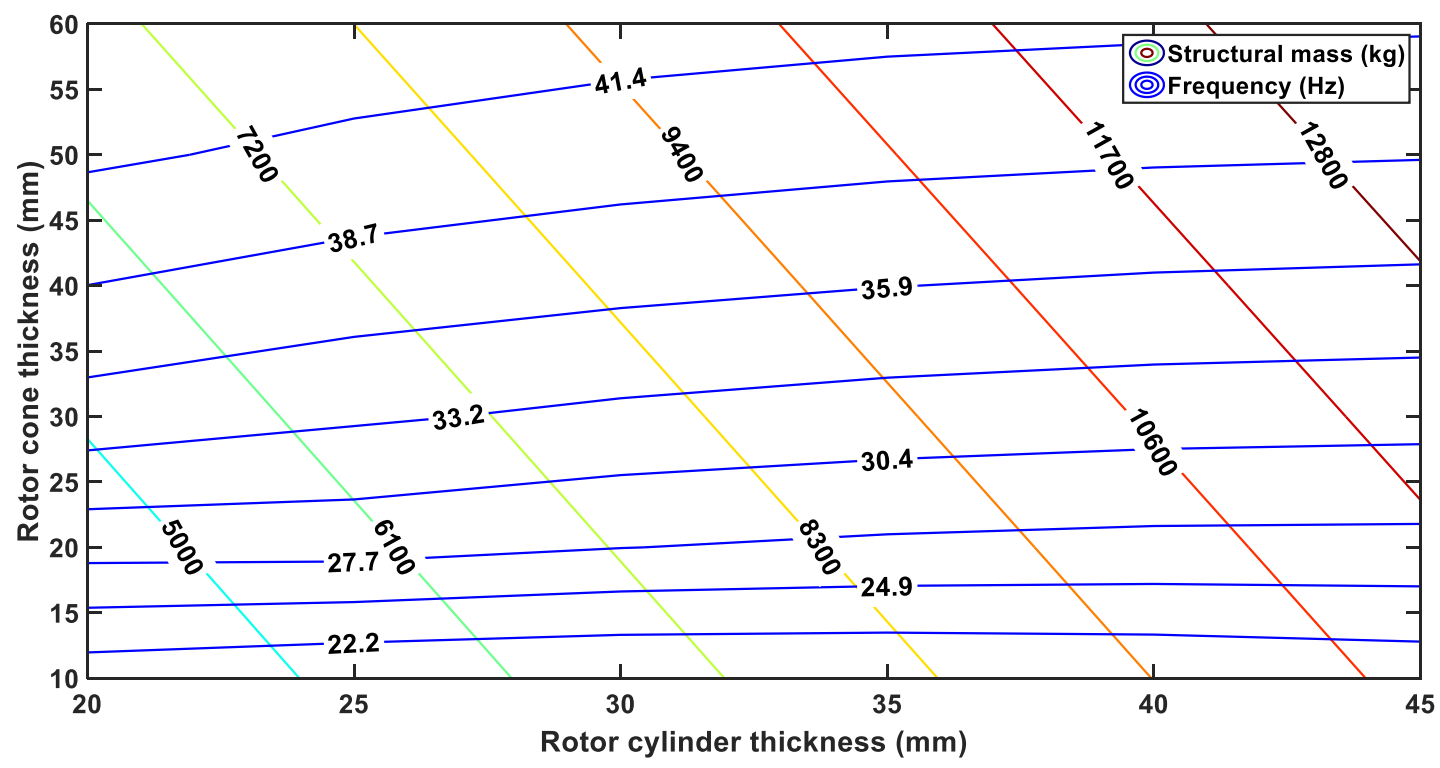

(b) 


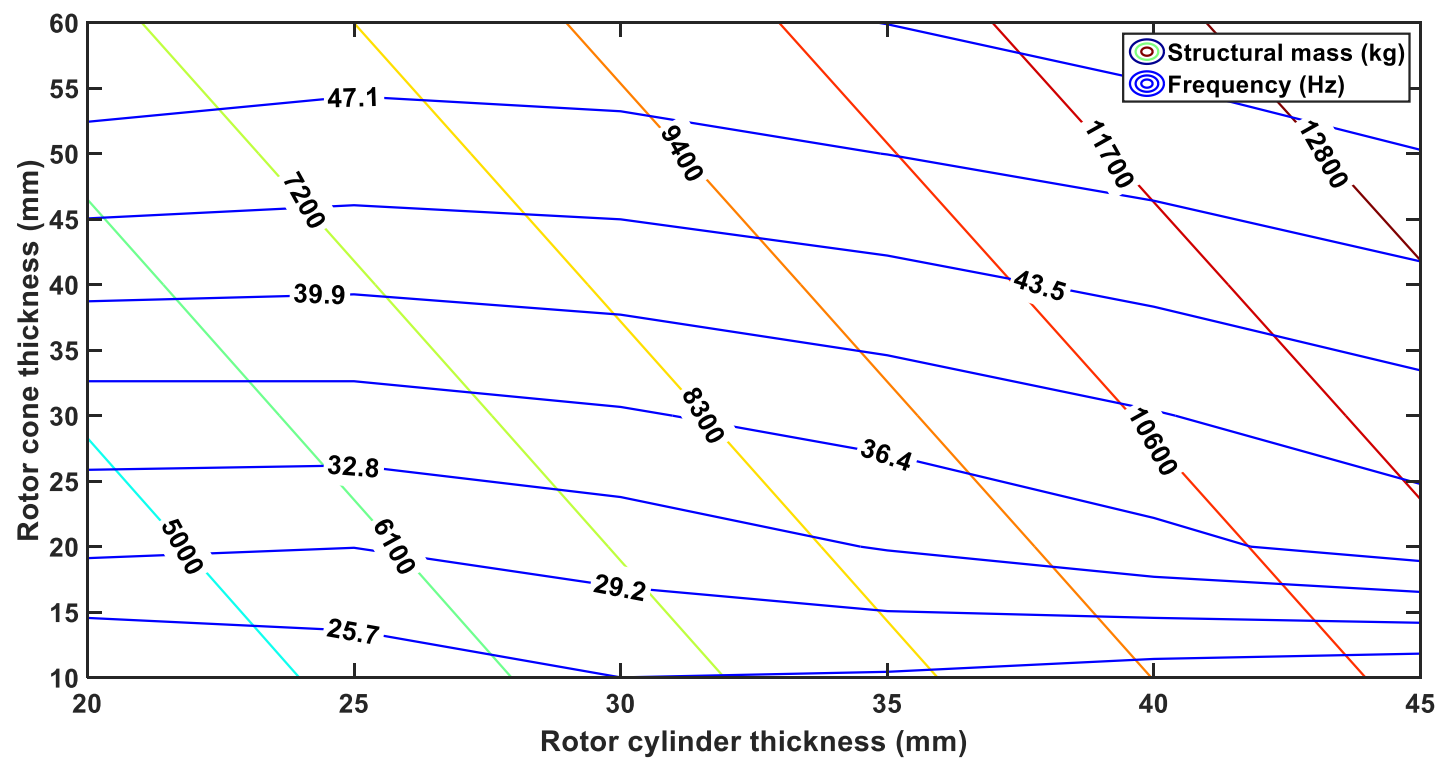

(c)

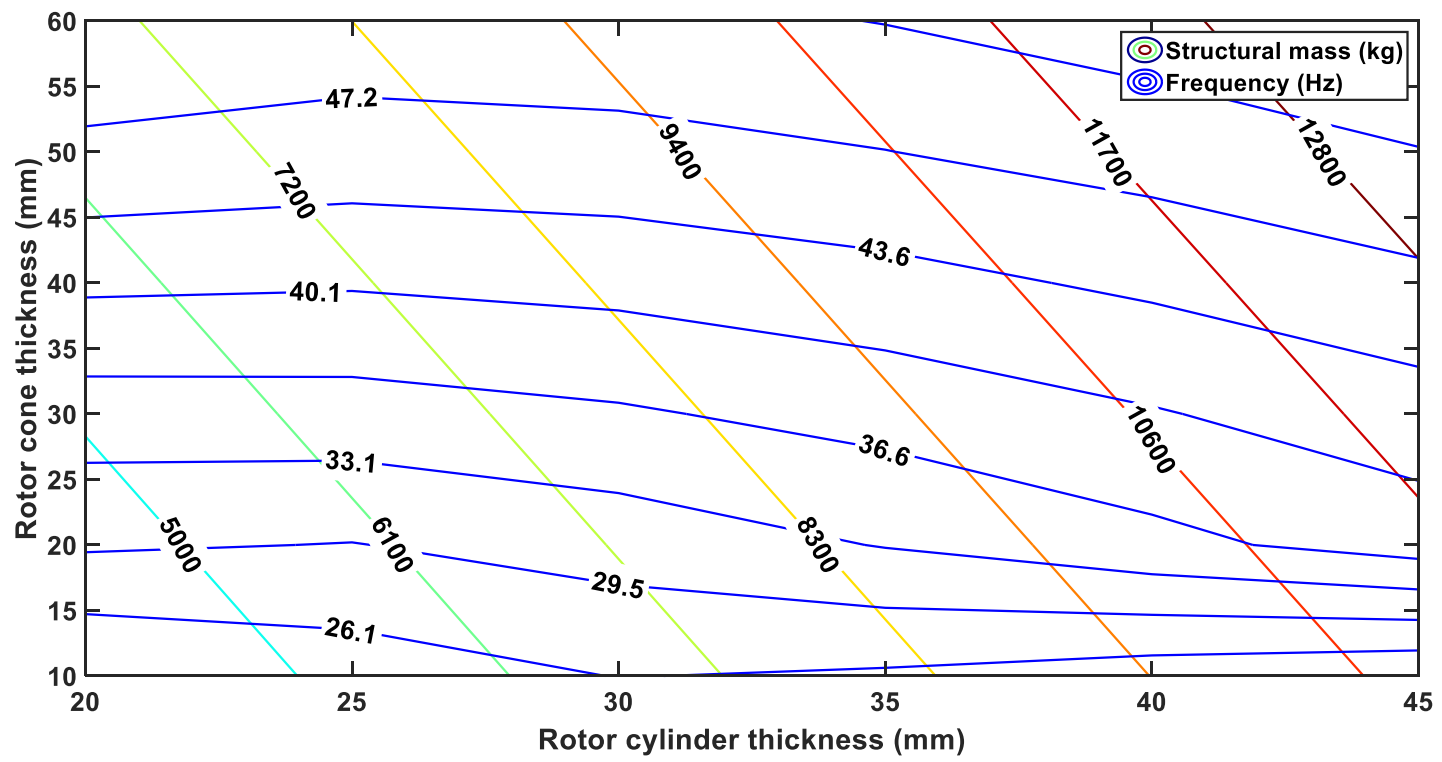

(d) 


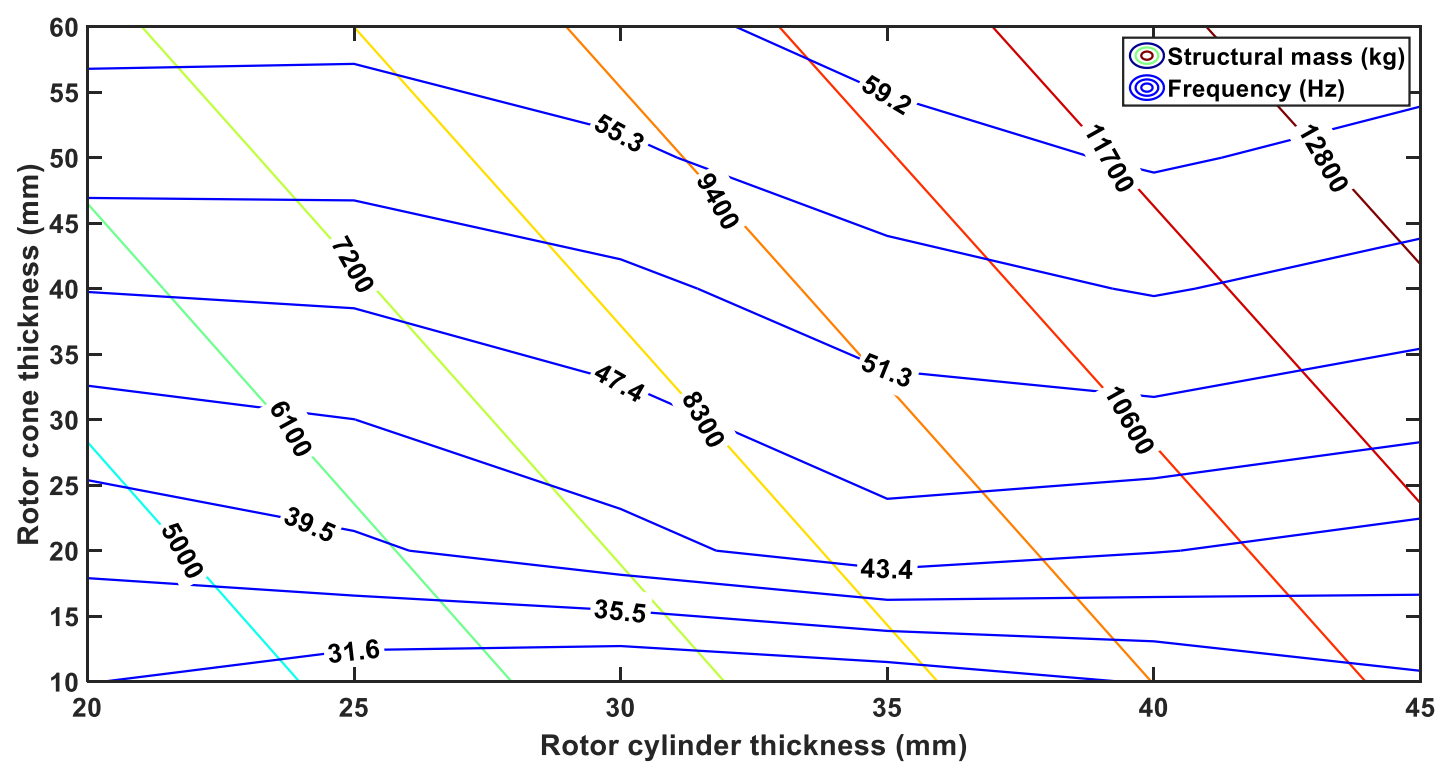

(e)

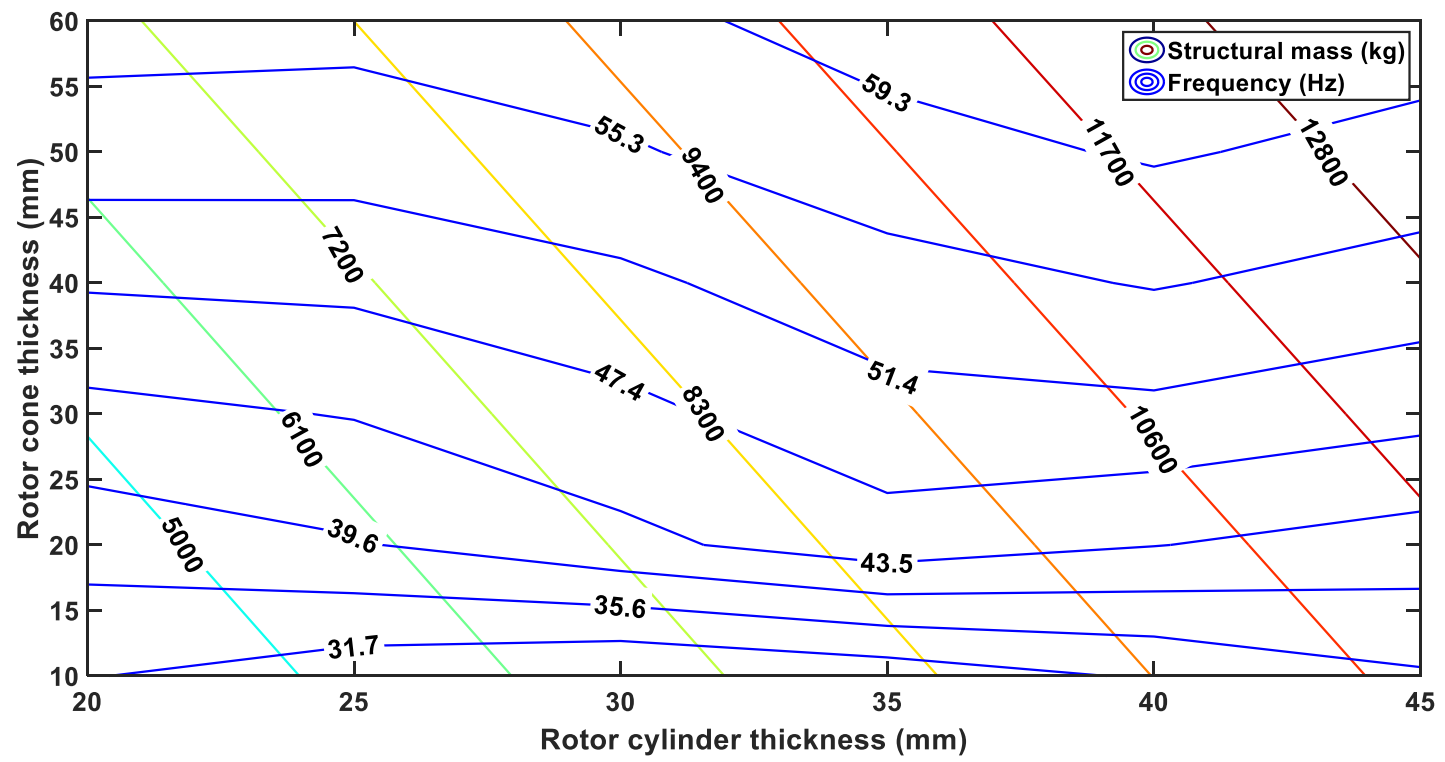

(f) 


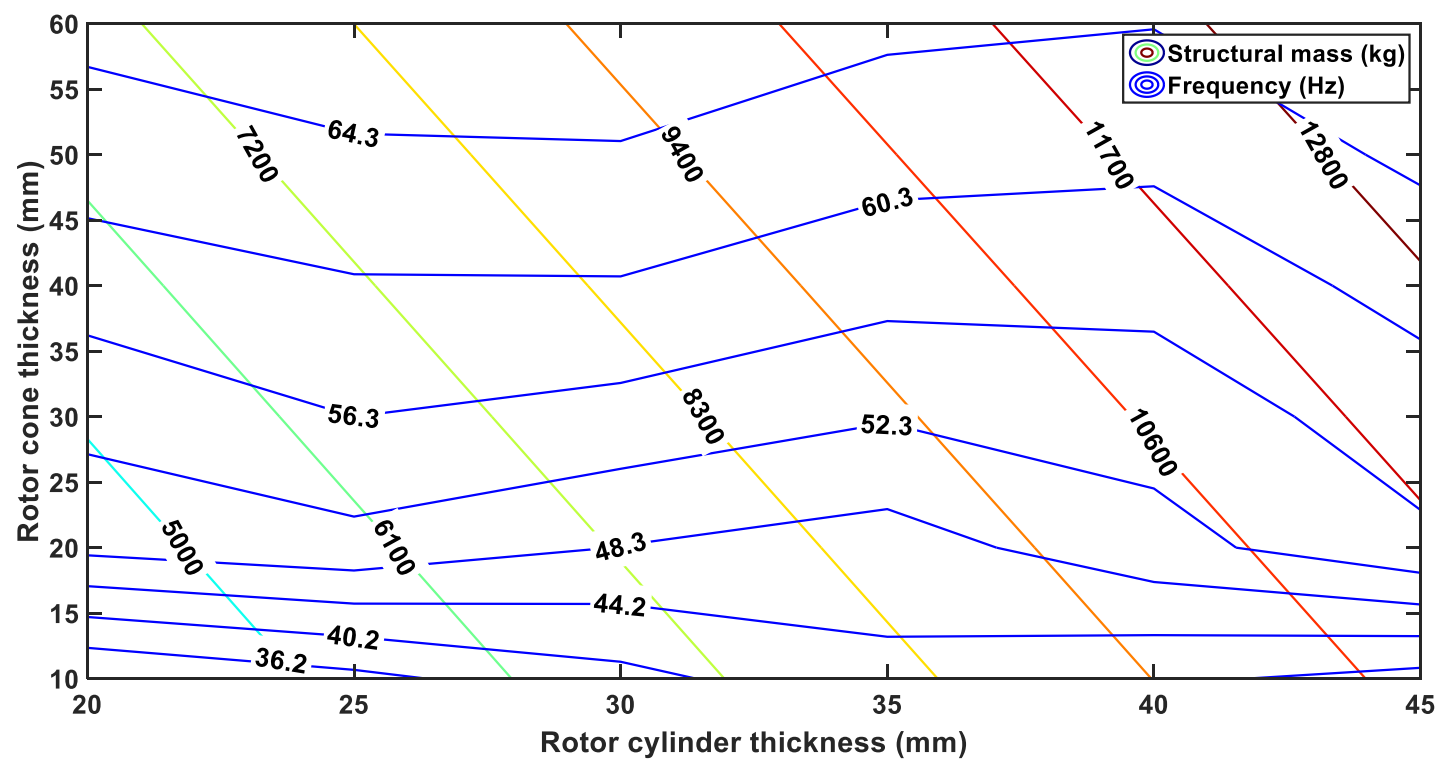

(g)

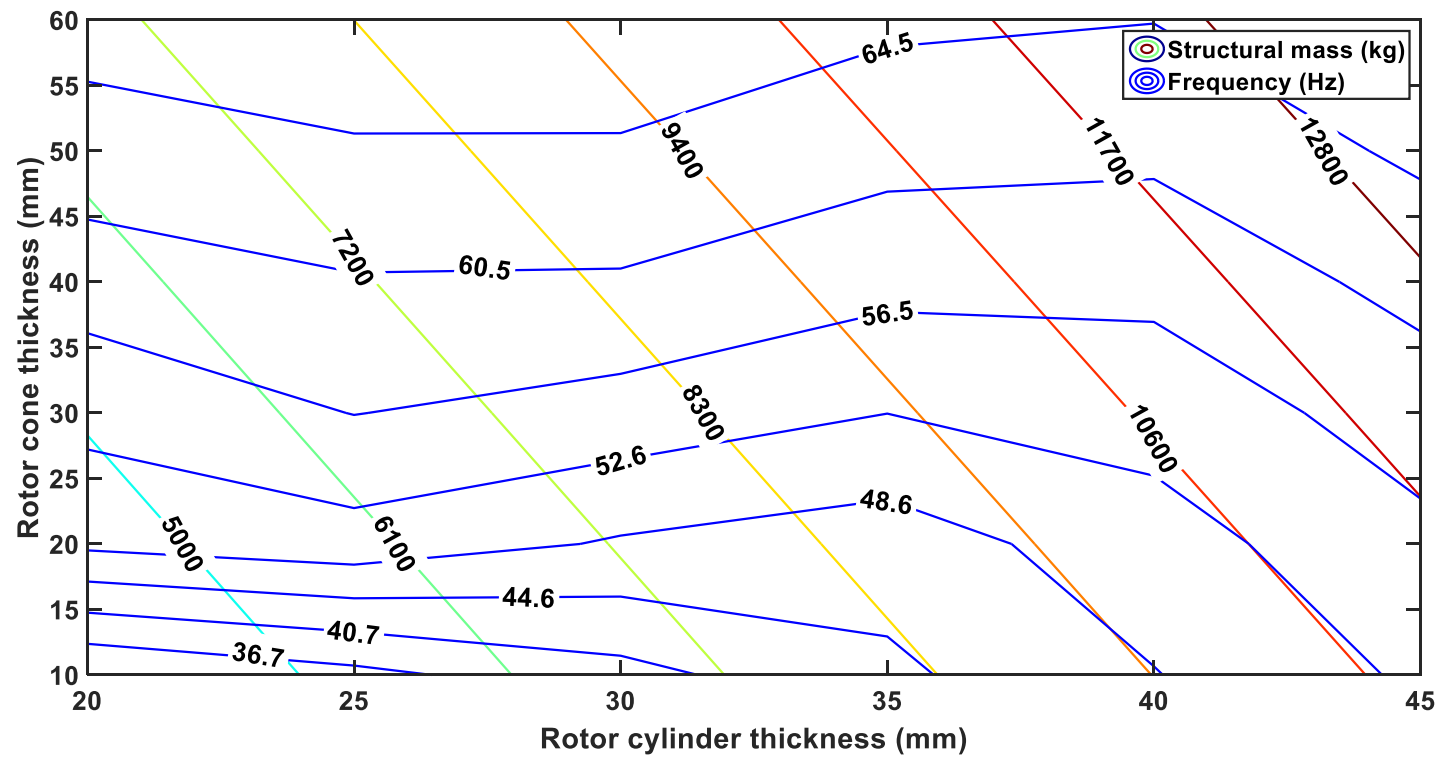

(h)

Figure A.1 2D Optimisation for $3 \mathrm{MW}$ rotor conical structures with natural frequencies criterion; (a) $1^{\text {st }}$ mode; (b) $2^{\text {nd }}$ mode; (c) $3^{\text {rd }}$ mode; (d) $4^{\text {th }}$ mode; (e) $5^{\text {th }}$ mode; (f) $6^{\text {th }}$ mode; (g) $7^{\text {th }}$ mode; (h) $8^{\text {th }}$ mode 